\title{
On the nonlinear pseudoparabolic equation with the mixed inhomogeneous condition
}

Le Thi Phuong Ngoc ${ }^{1}$, Truong Thi Nhan ${ }^{2,3}$, Tran Minh Thuyet ${ }^{4}$ and Nguyen Thanh Long ${ }^{3 *}$

\section{"Correspondence:}

longnt2@gmail.com

${ }^{3}$ Department of Mathematics and

Computer Science, University of

Natural Science, Vietnam National University Ho Chi Minh City,

227 Nguyen Van Cu Str., Dist. 5, Ho Chi Minh City, Vietnam

Full list of author information is available at the end of the article

\section{Abstract}

We study the following initial-boundary value problem:

$$
\left\{\begin{array}{l}
u_{t}-\left(\mu+\alpha \frac{\partial}{\partial t}\right)\left(\frac{\partial^{2} u}{\partial x^{2}}+\frac{1}{x} \frac{\partial u}{\partial x}\right)+f(u)=f_{1}(x, t), \quad 1<x<R, t>0 \\
u_{x}(1, t)=h_{1} u(1, t)+g_{1}(t), \quad u(R, t)=g_{R}(t) \\
u(x, 0)=\tilde{u}_{0}(x)
\end{array}\right.
$$

where $\mu>0, \alpha>0, h_{1} \geq 0, R>1$ are given constants and $f, f_{1}, g_{1}, g_{R}, \tilde{u}_{0}$ are given functions. First, we use the Galerkin and compactness method to prove the existence of a unique weak solution $u(t)$ of Problem (1) on $(0, T)$, for every $T>0$. Next, we study the asymptotic behavior of the solution $u(t)$ as $t \rightarrow+\infty$. Finally, we prove the existence and uniqueness of a weak solution of Problem (1) $)_{1,2}$ associated with a ' $(N+1)$-points condition in time' case,

$$
u(x, 0)=\sum_{i=1}^{N} \eta_{i} u\left(x, T_{i}\right)
$$

where $\left(T_{i}, \eta_{i}\right), i=1, \ldots, N$, are given constants satisfying

$$
0<T_{1}<T_{2}<\cdots<T_{N-1}<T_{N} \equiv T, \quad \sum_{i=1}^{N}\left|\eta_{i}\right| \leq 1
$$

MSC: 34B60; 35K55; 35Q72; 80Axx

Keywords: nonlinear pseudoparabolic equation; Faedo-Galerkin approximation; asymptotic behavior; ' $(N+1)$-points condition in time'

\section{Introduction}

Consider the following nonlinear pseudoparabolic equation:

$$
u_{t}-\left(\mu+\alpha \frac{\partial}{\partial t}\right)\left(\frac{\partial^{2} u}{\partial x^{2}}+\frac{1}{x} \frac{\partial u}{\partial x}\right)+f(u)=f_{1}(x, t), \quad 1<x<R, t>0,
$$

\section{Springer}


with the mixed inhomogeneous condition

$$
u_{x}(1, t)=h_{1} u(1, t)+g_{1}(t), \quad u(R, t)=g_{R}(t),
$$

and with the initial condition

$$
u(x, 0)=\tilde{u}_{0}(x)
$$

or the ' $(N+1)$-points condition in time' case

$$
u(x, 0)=\sum_{i=1}^{N} \eta_{i} u\left(x, T_{i}\right)
$$

where $\left(T_{i}, \eta_{i}\right), i=1, \ldots, N$, are given constants satisfying

$$
0<T_{1}<T_{2}<\cdots<T_{N-1}<T_{N} \equiv T, \quad \sum_{i=1}^{N}\left|\eta_{i}\right| \leq 1,
$$

here $\mu>0, \alpha>0, R>1, h_{1} \geq 0$ are given constants and $f, f_{1}, g_{1}, g_{R}, \tilde{u}_{0}$ are given functions satisfying conditions specified later.

The initial-boundary value problem (1.1)-(1.3) is classical and has a long history of applications and mathematical development. We refer to the monographs of Al'shin [1], and of Carroll and Showalter [2] for references and results on pseudoparabolic or Sobolev type equations. We also refer to [3] for asymptotic behavior and to [4] for nonlinear problems. Problems of this type arise in material science and physics, which have been extensively studied and several results concerning existence, regularity and asymptotic behavior have been established.

Equation (1.1) arises within the frameworks of mathematical models in engineering and physical sciences; see [5-13] and the references therein for interesting results on second grade fluids or a fourth grade fluid or other unsteady flows. It is well known that fluid solid mixtures are generally considered as second grade fluids and are modeled as fluids with variable physical parameters, thus, an analysis is performed for a second grade fluid with space dependent viscosity, elasticity and density.

In [9], some unsteady flow problems of a second grade fluid were considered. The flows are generated by the sudden application of a constant pressure gradient or by the impulsive motion of a boundary. Here, the velocities of the flows are described by the partial differential equations and exact analytic solutions of these differential equations are obtained. Suppose that the second grade fluid is in a circular cylinder and is initially at rest, and the fluid starts suddenly due to the motion of the cylinder parallel to its length. The axis of the cylinder is chosen as the $z$-axis. Using cylindrical polar coordinates, the governing partial differential equation is

$$
\left\{\begin{array}{l}
\frac{\partial w}{\partial t}=\left(v+\alpha \frac{\partial}{\partial t}\right)\left(\frac{\partial^{2}}{\partial r^{2}}+\frac{1}{r} \frac{\partial}{\partial r}\right) w(r, t)-N w \\
w(a, t)=W, \quad t>0 \\
w(r, 0)=0, \quad 0 \leq r<a
\end{array}\right.
$$

where $w(r, t)$ is the velocity along the $z$-axis, $v$ is the kinematic viscosity, $\alpha$ is the material parameter, and $N$ is the imposed magnetic field. Under the boundary and initial conditions, $W$ is the constant velocity at $r=a$ and $a$ is the radius of the cylinder. 
In [6], two types of time-dependent flows were investigated. An eigen function expansion method was used to find the velocity distribution. The obtained solutions satisfy the boundary and initial conditions and the governing equation. Remarkably some exact analytic solutions are possible for flows involving second grade fluid with variable material properties in terms of trigonometric and Chebyshev functions.

In [5], Mahmood et al. have considered the longitudinal oscillatory motion of a second grade fluid between two infinite coaxial circular cylinders, oscillating along their common axis with given constant angular frequencies $\Omega_{1}$ and $\Omega_{2}$. Velocity field and associated tangential stress of the motion were determined by using Laplace and Hankel transforms. In order to find exact analytic solutions for the flow of a second grade fluid between two longitudinally oscillating cylinders, the following problem was studied:

$$
\left\{\begin{array}{l}
\frac{\partial v}{\partial t}=\left(\mu+\alpha \frac{\partial}{\partial t}\right)\left(\frac{\partial^{2}}{\partial r^{2}}+\frac{1}{r} \frac{\partial}{\partial r}\right) v(r, t), \quad R_{1}<r<R_{2}, t>0 \\
v\left(R_{1}, t\right)=V_{1} \sin \left(\Omega_{1} t\right), \quad v\left(R_{2}, t\right)=V_{2} \sin \left(\Omega_{2} t\right) \\
u(r, 0)=0, \quad R_{1} \leq r \leq R_{2}
\end{array}\right.
$$

where $0<R_{1}<R_{2}, \mu, \alpha, V_{2}, \Omega_{1}, \Omega_{2}$ are positive constants. The solutions obtained have been presented in the series form in terms of Bessel functions $J_{0}(x), Y_{0}(x), J_{1}(x), Y_{1}(x)$, $J_{2}(x)$ and $Y_{2}(x)$, satisfying the governing equation and all imposed initial and boundary conditions.

The nonlinear parabolic problems of the form (1.1)-(1.3), with/without the term $\left(u_{r r}+\right.$ $\frac{\gamma}{r} u_{r}$ ), were also studied in $[14,15]$ and the references therein. In [14], by using the Galerkin and compactness method in appropriate Sobolev spaces with weight, the authors proved the existence of a unique weak solution of the following initial and boundary value problem for a nonlinear parabolic equation:

$$
\left\{\begin{array}{l}
u_{t}-a(t)\left(u_{r r}+\frac{\gamma}{r} u_{r}\right)+F(r, u)=f(r, t), \quad 0<r<1,0<t<T, \\
\left|\lim _{r \rightarrow 0_{+}} r^{\frac{\gamma}{2}} u_{r}(r, t)\right|<+\infty, \quad u_{r}(1, t)+h(t)\left(u(1, t)-\bar{u}_{0}\right)=0, \\
u(r, 0)=u_{0}(r) .
\end{array}\right.
$$

Furthermore, asymptotic behavior of the solution as $t \rightarrow+\infty$ was studied. In [15], the following nonlinear heat equation associated with Dirichlet-Robin conditions was investigated:

$$
\left\{\begin{array}{l}
u_{t}-\frac{\partial}{\partial x}\left[\mu(x, t) u_{x}\right]+f(u)=f_{1}(x, t),(x, t) \in \Omega \times(0, T), \\
u_{x}(0, t)=h_{0} u(0, t)+g_{0}(t), \quad-u_{x}(1, t)=h_{1} u(1, t)+g_{1}(t), \\
u(x, 0)=u_{0}(x) .
\end{array}\right.
$$

The condition (1.3a), which we call ' $(N+1)$-points condition in time', is known as a drifted periodic condition; see [16]. Indeed, if $u(t)=\sum_{i=1}^{N} \eta_{i} u\left(t+T_{i}\right)$, in the case of $0<\left|\eta_{N}\right| \leq 1$, then we have

$$
\begin{aligned}
u(t+T) & =\frac{1}{\eta_{N}}\left[u(t)-\sum_{i=1}^{N-1} \eta_{i} u\left(t+T_{i}\right)\right] \\
& =u(t)+\left(\frac{1}{\eta_{N}}-1\right) u(t)-\frac{1}{\eta_{N}} \sum_{i=1}^{N-1} \eta_{i} u\left(t+T_{i}\right), \quad \forall t \geq 0,
\end{aligned}
$$


it means

$$
u(t+T)=u(t)+\delta(t), \quad \forall t \geq 0,
$$

with $\delta(t)=\left(\frac{1}{\eta_{N}}-1\right) u(t)-\frac{1}{\eta_{N}} \sum_{i=1}^{N-1} \eta_{i} u\left(t+T_{i}\right)$ satisfying the condition

$$
\delta(t)=\sum_{i=1}^{N} \eta_{i} \delta\left(t+T_{i}\right), \quad \forall t \geq 0
$$

Note that (1.10) holds by the fact that

$$
\begin{aligned}
\sum_{j=1}^{N} \eta_{j} \delta\left(t+T_{j}\right) & =\sum_{j=1}^{N} \eta_{j}\left[\left(\frac{1}{\eta_{N}}-1\right) u\left(t+T_{j}\right)-\frac{1}{\eta_{N}} \sum_{i=1}^{N-1} \eta_{i} u\left(t+T_{i}+T_{j}\right)\right] \\
& =\left(\frac{1}{\eta_{N}}-1\right) \sum_{j=1}^{N} \eta_{j} u\left(t+T_{j}\right)-\frac{1}{\eta_{N}} \sum_{i=1}^{N-1} \eta_{i} \sum_{j=1}^{N} \eta_{j} u\left(t+T_{i}+T_{j}\right) \\
& =\left(\frac{1}{\eta_{N}}-1\right) u(t)-\frac{1}{\eta_{N}} \sum_{i=1}^{N-1} \eta_{i} u\left(t+T_{i}\right)=\delta(t), \quad \forall t \geq 0
\end{aligned}
$$

With $\eta_{1}=\eta_{2}=\cdots=\eta_{N-1}=0, \eta_{N}=1$, (1.3a) leads to the $T$-periodic condition

$$
u(x, 0)=u(x, T)
$$

and with $\eta_{1}=\eta_{2}=\cdots=\eta_{N-1}=0, \eta_{N}=-1$, we have the anti-periodic condition

$$
u(x, 0)=-u(x, T)
$$

The present paper is concerned with the second grade fluid in a circular cylinder associated with the initial condition (1.3) or the drifted periodic condition (1.9). The extensive study of such flows is motivated by both their fundamental interest and their practical importance; see [9]. The arrangement of the paper is as follows. In Section 2, we present preliminaries. In Section 3, under appropriate conditions, we prove the existence of a unique weak solution of Problem (1.1)-(1.3). In Section 4, we consider asymptotic behavior of the solution of Problem (1.1)-(1.3), as $t \rightarrow+\infty$. Finally, in Section 5, we establish the existence and uniqueness of a weak solution of Problem (1.1), (1.2), (1.3a).

Because of the mathematical context, the results obtained here generalize relative to the ones in $[14,15]$, by using the same techniques and with some appropriate modifications. On the other hand, the fixed point method is also applied.

\section{Preliminaries}

Put $\Omega=(1, R), Q_{T}=\Omega \times(0, T), T>0$. We omit the definitions of the usual function spaces: $C^{m}(\bar{\Omega}), L^{p}(\Omega), W^{m, p}(\Omega)$. We define $W^{m, p}=W^{m, p}(\Omega), L^{p}=W^{0, p}(\Omega), H^{m}=W^{m, 2}(\Omega), 1 \leq$ $p \leq \infty, m=0,1, \ldots$. The norm in $L^{2}$ is denoted by $\|\cdot\|$. We also denote by $(\cdot, \cdot)$ the scalar product in $L^{2}$. We denote by $\|\cdot\|_{X}$ the norm of a Banach space $X$ and by $X^{\prime}$ the dual 
space of $X$. We denote by $L^{p}(0, T ; X), 1 \leq p \leq \infty$, the Banach space of the real functions $u:(0, T) \rightarrow X$ measurable, such that

$$
\|u\|_{L^{p}(0, T ; X)}=\left(\int_{0}^{T}\|u(t)\|_{X}^{p} d t\right)^{1 / p}<\infty \quad \text { for } 1 \leq p<\infty
$$

and

$$
\|u\|_{L^{\infty}(0, T ; X)}=\underset{0<t<T}{\operatorname{ess} \sup }\|u(t)\|_{X} \quad \text { for } p=\infty .
$$

On $H^{1}$, we shall use the following norm:

$$
\|v\|_{H^{1}}=\left(\|v\|^{2}+\left\|v_{x}\right\|^{2}\right)^{1 / 2} .
$$

We put

$$
V=\left\{v \in H^{1}: v(R)=0\right\} .
$$

$V$ is a closed subspace of $H^{1}$ and on $V$ two norms $\|v\|_{H^{1}}$ and $\left\|v_{x}\right\|$ are equivalent norms. Note that $L^{2}, H^{1}$ are also the Hilbert spaces with the corresponding scalar products

$$
\langle u, v\rangle=\int_{1}^{R} x u(x) v(x) d x, \quad\langle u, v\rangle+\left\langle u_{x}, v_{x}\right\rangle
$$

respectively. The norms in $L^{2}$ and $H^{1}$ induced by the corresponding scalar products are denoted by $\|\cdot\|_{0}$ and $\|\cdot\|_{1}$, respectively. $V$ is continuously and densely embedded in $L^{2}$. Identifying $L^{2}$ with $\left(L^{2}\right)^{\prime}$ (the dual of $L^{2}$ ), we have $V \hookrightarrow L^{2} \hookrightarrow V^{\prime}$; On the other hand, the notation $\langle\cdot, \cdot\rangle$ is used for the pairing between $V$ and $V^{\prime}$.

We then have the following lemmas, the proofs of which can be found in [17].

Lemma 2.1 We have the following inequalities:

(i) $\|v\| \leq\|v\|_{0} \leq \sqrt{R}\|v\| \quad$ for all $v \in L^{2}$,

(ii) $\|v\|_{H^{1}} \leq\|v\|_{1} \leq \sqrt{R}\|v\|_{H^{1}} \quad$ for all $v \in H^{1}$.

Lemma 2.2 The imbedding $H^{1} \hookrightarrow C^{0}(\bar{\Omega})$ is compact.

Lemma 2.3 The imbedding $V \hookrightarrow C^{0}(\bar{\Omega})$ is compact and

(i) $\|v\|_{C^{0}(\bar{\Omega})} \leq \sqrt{R-1}\left\|v_{x}\right\| \quad$ for all $v \in V$,

(ii) $\|v\| \leq \frac{R-1}{\sqrt{2}}\left\|v_{x}\right\| \quad$ for all $v \in V$,

(iii) $\|v\|_{0} \leq \sqrt{\frac{R}{2}}(R-1)\left\|v_{x}\right\|_{0} \quad$ for all $v \in V$.

Remark 2.1 On $L^{2}$, two norms $v \longmapsto\|v\|$ and $v \longmapsto\|v\|_{0}$ are equivalent. So are two norms $v \longmapsto\|v\|_{H^{1}}$ and $v \longmapsto\|v\|_{1}$ on $H^{1}$, and four norms $v \longmapsto\|v\|_{H^{1}}, v \longmapsto\|v\|_{1}, v \longmapsto\left\|v_{x}\right\|$ and $v \longmapsto\left\|v_{x}\right\|_{0}$ on $V$. 
Consider $a(\cdot, \cdot)$ is the symmetric bilinear form on $V \times V$ defined by

$$
a(u, w)=\left\langle u_{x}, w_{x}\right\rangle+h_{1} u(1) w(1) \quad \text { for all } u, w \in V,
$$

with $h_{1} \geq 0$ is given constant.

Then the symmetric bilinear form $a(\cdot, \cdot)$ is continuous on $V \times V$ and coercive on $V$.

We have also the following lemma.

Lemma 2.4 There exists the Hilbert orthonormal base $\left\{w_{j}\right\}$ of $L^{2}$ consisting of the eigenfunctions $w_{j}$ corresponding to the eigenvalue $\bar{\lambda}_{j}$ such that

$$
\left\{\begin{array}{l}
0<\bar{\lambda}_{1} \leq \bar{\lambda}_{2} \leq \cdots \leq \bar{\lambda}_{j} \leq \bar{\lambda}_{j+1} \leq \cdots, \quad \lim _{j \rightarrow+\infty} \bar{\lambda}_{j}=+\infty \\
a\left(w_{j}, w\right)=\bar{\lambda}_{j}\left\langle w_{j}, w\right\rangle \quad \text { for all } w \in V, j=1,2, \ldots
\end{array}\right.
$$

Furthermore, the sequence $\left\{w_{j} / \sqrt{\bar{\lambda}_{j}}\right\}$ is also the Hilbert orthonormal base of $V$ with respect to the scalar product $a(\cdot, \cdot)$.

On the other hand, we have $w_{j}$ satisfying the following boundary value problem:

$$
\left\{\begin{array}{l}
-\left(w_{j x x}+\frac{1}{x} w_{j x}\right)=\bar{\lambda}_{j} w_{j} \quad \text { in }(1, R) \\
w_{j x}(1)-h_{1} w_{j}(1)=w_{j}(R)=0, \quad w_{j} \in C^{\infty}([1, R]) .
\end{array}\right.
$$

The proof of Lemma 2.4 can be found in [18], p.87, Theorem 7.7, with $H=L^{2}$ and $a(\cdot, \cdot)$ as defined by (2.6).

\section{The existence and the uniqueness}

Now, we shall consider Problem (1.1)-(1.3) with $\alpha>0, \mu>0, h_{1} \geq 0$ are constants and make the following assumptions:

$\left(\mathrm{H}_{1}\right) \quad \tilde{u}_{0} \in V$;

$\left(\mathrm{H}_{2}\right) g_{1}, g_{R} \in W^{1,1}(0, T), \tilde{u}_{0 x}(1)-h_{1} \tilde{u}_{0}(1)=g_{1}(0), \tilde{u}_{0}(R)=g_{R}(0)$;

$\left(\mathrm{H}_{3}\right) f_{1} \in L^{1}\left(0, T ; L^{2}\right)$;

$\left(\mathrm{H}_{4}\right) f \in C^{0}(\mathbb{R} ; \mathbb{R})$ satisfies the condition that there exists positive constant $\delta$ such that

$$
(y-z)(f(y)-f(z)) \geq-\delta|y-z|^{2} \quad \text { for all } y, z \in \mathbb{R}
$$

In the case $g_{1} \neq 0$ or $g_{R} \neq 0$, it is clearly that Problem (1.1)-(1.3) reduces to a problem with homogeneous boundary conditions by the suitable transformation. Indeed, put

$$
\varphi(x, t)=\frac{(x-R) g_{1}(t)+\left[h_{1}(x-1)+1\right] g_{R}(t)}{1+(R-1) h_{1}} .
$$

By the transformation $v(x, t)=u(x, t)-\varphi(x, t)$, Problem (1.1)-(1.3) becomes the following problem:

$$
\left\{\begin{array}{l}
v_{t}-\left(\mu+\alpha \frac{\partial}{\partial t}\right)\left(\frac{\partial^{2} v}{\partial x^{2}}+\frac{1}{x} \frac{\partial v}{\partial x}\right)+f(v+\varphi)=f_{2}(x, t), \quad 1<x<R, t>0 \\
v_{x}(1, t)-h_{1} v(1, t)=v(R, t)=0 \\
v(x, 0)=\tilde{v}_{0}(x)
\end{array}\right.
$$


where

$$
\left\{\begin{aligned}
f_{2}(x, t)= & f_{1}(x, t)-\frac{1}{1+(R-1) h_{1}}\left[(x-R) g_{1}^{\prime}(t)+\left[h_{1}(x-1)+1\right] g_{R}^{\prime}(t)\right] \\
& \quad+\frac{1}{1+(R-1) h_{1}}\left[\frac{\mu}{x}\left(g_{1}(t)+h_{1} g_{R}(t)\right)+\frac{\alpha}{x}\left(g_{1}^{\prime}(t)+h_{1} g_{R}^{\prime}(t)\right)\right], \\
\tilde{v}_{0}(x)= & \tilde{u}_{0}(x)-\varphi(x, 0),
\end{aligned}\right.
$$

and $\tilde{u}_{0}, g_{1}, g_{R}$ satisfying the condition $\tilde{u}_{0 x}(1)-h_{1} \tilde{u}_{0}(1)=g_{1}(0), \tilde{u}_{0}(R)=g_{R}(0)$.

Remark 3.1 The weak formulation of the initial-boundary value problem (3.1) can be given in the following manner: Find $v \in L^{\infty}(0, T ; V)$ with $t v_{t} \in L^{2}(0, T ; V)$, such that $v$ satisfies the following variational equation:

$$
\left\{\begin{array}{l}
\frac{d}{d t}[\langle v(t), w\rangle+\alpha a(v(t), w)]+\mu a(v(t), w)+\langle f(v(t)+\varphi(t)), w\rangle \\
\quad=\left\langle f_{2}(t), w\right\rangle \text { for all } w \in V, \text { a.e., } t \in(0, T) \\
v(0)=\tilde{v}_{0}
\end{array}\right.
$$

where $a(\cdot, \cdot)$ is the symmetric bilinear form on $V \times V$ defined by (2.6).

Then we have the following theorem.

Theorem 3.1 Let $T>0$ and $\left(\mathrm{H}_{1}\right)-\left(\mathrm{H}_{4}\right)$ hold. Then Problem (3.1) has a unique weak solution $v$ such that

$$
v \in L^{\infty}(0, T ; V) \text { and } t v_{t} \in L^{2}(0, T ; V)
$$

Moreover, if $\left(\mathrm{H}_{3}\right)$ is replaced by $f_{1} \in L^{2}\left(Q_{T}\right)$, then the solution $v$ satisfies

$$
v \in L^{\infty}(0, T ; V) \text { and } v_{t} \in L^{2}(0, T ; V) .
$$

Proof The proof consists of several steps.

Step 1. The Faedo-Galerkin approximation (introduced by Lions [19]).

Consider the basis $\left\{w_{j}\right\}$ for $V$ as in Lemma 2.4. We find the approximate solution of Problem (3.1) in the form

$$
v_{m}(t)=\sum_{j=1}^{m} c_{m j}(t) w_{j}
$$

where the coefficients $c_{m j}$ satisfy the system of nonlinear differential equations

$$
\left\{\begin{array}{l}
\left\langle v_{m}^{\prime}(t), w_{j}\right\rangle+\alpha a\left(v_{m}^{\prime}(t), w_{j}\right)+\mu a\left(v_{m}(t), w_{j}\right)+\left\langle f\left(v_{m}(t)+\varphi(t)\right), w_{j}\right\rangle \\
\quad=\left\langle f_{2}(t), w_{j}\right\rangle, \quad 1 \leq j \leq m \\
v_{m}(0)=v_{0 m}
\end{array}\right.
$$

and

$$
v_{0 m}=\sum_{j=1}^{m} \alpha_{m j} w_{j} \rightarrow \tilde{v}_{0} \quad \text { strongly in } V .
$$


The system of equations (3.7) can be rewritten in form

$$
\left\{\begin{array}{l}
c_{m j}^{\prime}(t)+\frac{\mu \bar{\lambda}_{j}}{1+\alpha \bar{\lambda}_{j}} c_{m j}(t)+\frac{1}{1+\alpha \bar{\lambda}_{j}}\left\langle f\left(v_{m}(t)+\varphi(t)\right), w_{j}\right\rangle=\frac{1}{1+\alpha \bar{\lambda}_{j}}\left\langle f_{2}(t), w_{j}\right\rangle, \\
c_{m}(0)=\alpha_{m j}, \quad 1 \leq j \leq m .
\end{array}\right.
$$

It is clear that for each $m$ there exists a solution $v_{m}(t)$ in the form (3.6) which satisfies (3.7) almost everywhere on $0 \leq t \leq \tilde{T}_{m}$ for some $\tilde{T}_{m}, 0<\tilde{T}_{m} \leq T$. The following estimates allow one to take $\tilde{T}_{m}=T$ for all $m$.

Step 2. A priori estimates.

(a) The first estimate. Multiplying the $j$ th equation of (3.7) by $c_{m j}(t)$ and summing up with respect to $j$, afterward, integrating by parts with respect to the time variable from 0 to $t$, we get after some rearrangements

$$
\begin{gathered}
\left\|v_{m}(t)\right\|_{0}^{2}+\alpha a\left(v_{m}(t), v_{m}(t)\right)+2 \mu \int_{0}^{t} a\left(v_{m}(s), v_{m}(s)\right) d s+2 \int_{0}^{t}\left\langle f\left(v_{m}(s)+\varphi(s)\right), v_{m}(s)\right\rangle d s \\
=\left\|v_{0 m}\right\|_{0}^{2}+\alpha a\left(v_{0 m}, v_{0 m}\right)+2 \int_{0}^{t}\left\langle f_{2}(s), v_{m}(s)\right\rangle d s .
\end{gathered}
$$

By $v_{0 m} \rightarrow \tilde{v}_{0}$ strongly in $V$, we have

$$
\left\|v_{0 m}\right\|_{0}^{2}+\alpha a\left(v_{0 m}, v_{0 m}\right) \leq C_{0} \quad \text { for all } m
$$

where $C_{0}$ always indicates a bound depending on $\tilde{v}_{0}$.

By the assumptions $\left(\mathrm{H}_{4}\right)$, and with $\varepsilon_{1}>0$, we estimate without difficulty the following terms in (3.10):

$$
\begin{aligned}
& 2 \int_{0}^{t}\left\langle f\left(v_{m}(s)+\varphi(s)\right), v_{m}(s)\right\rangle d s \\
& \quad=2 \int_{0}^{t}\left\langle f\left(v_{m}(s)+\varphi(s)\right)-f(\varphi(s)), v_{m}(s)\right\rangle d s+2 \int_{0}^{t}\left\langle f(\varphi(s)), v_{m}(s)\right\rangle d s \\
& \quad \geq-2 \delta \int_{0}^{t}\left\|v_{m}(s)\right\|_{0}^{2} d s-2 \int_{0}^{t}\|f(\varphi(s))\|_{0}\left\|v_{m}(s)\right\|_{0} d s \\
& \quad \geq-\left(2 \delta+\varepsilon_{1}\right) \int_{0}^{t}\left\|v_{m}(s)\right\|_{0}^{2} d s-\frac{1}{\varepsilon_{1}} \int_{0}^{T}\|f(\varphi(s))\|_{0}^{2} d s \\
& 2 \int_{0}^{t}\left\langle f_{2}(s), v_{m}(s)\right\rangle d s \leq\left\|f_{2}\right\|_{L^{1}\left(0, T ; L^{2}\right)}+\int_{0}^{t}\left\|f_{2}(s)\right\|_{0}\left\|v_{m}(s)\right\|_{0}^{2} d s .
\end{aligned}
$$

Hence, it follows from (3.10)-(3.13) that

$$
S_{m}(t) \leq C_{T}^{(1)}+\int_{0}^{t} C_{T}^{(2)}(s) S_{m}(s) d s
$$

where

$$
\left\{\begin{array}{l}
S_{m}(t)=\left\|v_{m}(t)\right\|_{0}^{2}+\alpha a\left(v_{m}(t), v_{m}(t)\right)+2 \mu \int_{0}^{t} a\left(v_{m}(s), v_{m}(s)\right) d s \\
C_{T}^{(1)}=C_{0}+\left\|f_{2}\right\|_{L^{1}\left(0, T ; L^{2}\right)}+\frac{1}{\varepsilon_{1}} \int_{0}^{T}\|f(\varphi(s))\|_{0}^{2} d s \\
C_{T}^{(2)}(s)=2 \delta+\varepsilon_{1}+\left\|f_{2}(s)\right\|_{0}, \quad C_{T}^{(2)} \in L^{1}(0, T) .
\end{array}\right.
$$


By Gronwall's lemma, we obtain from (3.14)

$$
S_{m}(t) \leq C_{T}^{(1)} \exp \left(\int_{0}^{t} C_{T}^{(2)}(s) d s\right) \leq C_{T}
$$

for all $m \in \mathbb{N}$, for all $t, 0 \leq t \leq \tilde{T}_{m} \leq T$, i.e., $\tilde{T}_{m}=T$, where $C_{T}$ always indicates a bound depending on $T$.

(b) The second estimate. Multiplying the $j$ th equation of the system (3.7) by $2 t^{2} c_{m j}^{\prime}(t)$ and summing up with respect to $j$, we have

$$
\begin{aligned}
& 2\left\|t v_{m}^{\prime}(t)\right\|_{0}^{2}+2 \alpha a\left(t v_{m}^{\prime}(t), t v_{m}^{\prime}(t)\right)+\mu \frac{d}{d t} a\left(t v_{m}(t), t v_{m}(t)\right) \\
& =2 \mu t a\left(v_{m}(t), v_{m}(t)\right)-2\left\langle t f\left(v_{m}(t)+\varphi(t)\right), t v_{m}^{\prime}(t)\right\rangle+2\left\langle t f_{2}(t), t v_{m}^{\prime}(t)\right\rangle .
\end{aligned}
$$

Integrating (3.17), we get

$$
\begin{aligned}
2 \int_{0}^{t}\left\|s v_{m}^{\prime}(s)\right\|_{0}^{2} d s+2 \alpha \int_{0}^{t} a\left(s v_{m}^{\prime}(s), s v_{m}^{\prime}(s)\right) d s+\mu a\left(t v_{m}(t), t v_{m}(t)\right) \\
=2 \mu \int_{0}^{t} s a\left(v_{m}(s), v_{m}(s)\right) d s-2 \int_{0}^{t}\left\langle s f\left(v_{m}(s)+\varphi(s)\right), s v_{m}^{\prime}(s)\right\rangle d s \\
\quad+2 \int_{0}^{t}\left\langle s f_{2}(s), s v_{m}^{\prime}(s)\right\rangle d s .
\end{aligned}
$$

We shall estimate the terms of (3.18) as follows:

$$
\begin{aligned}
2 \mu \int_{0}^{t} s a\left(v_{m}(s), v_{m}(s)\right) d s \leq 2 \mu T \int_{0}^{t} a\left(v_{m}(s), v_{m}(s)\right) d s \leq T S_{m}(t) \leq C_{T} \\
2 \int_{0}^{t}\left\langle s f_{2}(s), s v_{m}^{\prime}(s)\right\rangle d s \leq 2 \int_{0}^{t}\left\|s f_{2}(s)\right\|_{0}^{2} d s+\frac{1}{2} \int_{0}^{t}\left\|s v_{m}^{\prime}(s)\right\|_{0}^{2} d s \\
\leq C_{T}+\frac{1}{2} \int_{0}^{t}\left\|s v_{m}^{\prime}(s)\right\|_{0}^{2} d s
\end{aligned}
$$

Note that

$$
\begin{aligned}
|\varphi(x, t)| & \leq \frac{(R-1)}{1+(R-1) h_{1}}\left|g_{1}(t)\right|+\frac{h_{1}(R-1)+1}{1+(R-1) h_{1}}\left|g_{R}(t)\right| \\
& \leq\left[R+h_{1}(R-1)\right]\left[\left|g_{1}(t)\right|+\left|g_{R}(t)\right|\right],
\end{aligned}
$$

hence

$$
\begin{aligned}
\left|v_{m}(x, s)\right|+|\varphi(x, s)| & \leq\left\|v_{m}(s)\right\|_{C^{0}(\bar{\Omega})}+\left[R+h_{1}(R-1)\right]\left(\left|g_{1}(s)\right|+\left|g_{R}(s)\right|\right) \\
& \leq \sqrt{R-1} \sqrt{\frac{S_{m}(s)}{\alpha}}+\left[R+h_{1}(R-1)\right]\left(\left\|g_{1}\right\|_{C^{0}([0, T])}+\left\|g_{R}\right\|_{C^{0}([0, T])}\right) \\
& \leq \sqrt{\frac{(R-1) C_{T}}{\alpha}}+\left[R+h_{1}(R-1)\right]\left(\left\|g_{1}\right\|_{C^{0}([0, T])}+\left\|g_{R}\right\|_{C^{0}([0, T])}\right) \\
& \equiv \bar{C}_{T} .
\end{aligned}
$$


This implies

$$
\begin{aligned}
2 \int_{0}^{t}\left\langle s f\left(v_{m}(s)+\varphi(s)\right), s v_{m}^{\prime}(s)\right\rangle d s & \leq 2 \int_{0}^{t}\left\|s f\left(v_{m}(s)+\varphi(s)\right)\right\|_{0}^{2} d s+\frac{1}{2} \int_{0}^{t}\left\|s v_{m}^{\prime}(s)\right\|_{0}^{2} d s \\
& \leq 2 \int_{0}^{t} s^{2} \int_{1}^{R} x \sup _{|z| \leq \bar{C}_{T}} f^{2}(z) d x d s+\frac{1}{2} \int_{0}^{t}\left\|s v_{m}^{\prime}(s)\right\|_{0}^{2} d s \\
& \leq C_{T}+\frac{1}{2} \int_{0}^{t}\left\|s v_{m}^{\prime}(s)\right\|_{0}^{2} d s
\end{aligned}
$$

It follows from (3.18)-(3.20) and (3.22) that

$$
\int_{0}^{t}\left\|s v_{m}^{\prime}(s)\right\|_{0}^{2} d s+2 \alpha \int_{0}^{t} a\left(s v_{m}^{\prime}(s), s v_{m}^{\prime}(s)\right) d s+\mu a\left(t v_{m}(t), t v_{m}(t)\right) \leq 3 C_{T}
$$

for all $m \in \mathbb{N}$, for all $t \in[0, T], \forall T>0$, where $C_{T}$ always indicates a bound depending on $T$.

By $\left(t v_{m x}\right)^{\prime}=t v_{m x}^{\prime}+v_{m x}$ and (3.16) we deduce that

$$
\begin{aligned}
\left\|\left(t v_{m x}\right)^{\prime}\right\|_{L^{2}\left(Q_{T}\right)} & \leq\left\|t v_{m x}^{\prime}\right\|_{L^{2}\left(Q_{T}\right)}+\left\|v_{m x}\right\|_{L^{2}\left(Q_{T}\right)} \\
& \leq \sqrt{\int_{0}^{T} a\left(s v_{m}^{\prime}(s), s v_{m}^{\prime}(s)\right) d s}+\sqrt{T}\left\|v_{m}\right\|_{L^{\infty}(0, T ; V)} \leq C_{T} .
\end{aligned}
$$

\section{Step 3. The limiting process.}

By (3.16), (3.23) and (3.24) we deduce that there exists a subsequence of $\left\{v_{m}\right\}$, still denoted by $\left\{v_{m}\right\}$ such that

$$
\left\{\begin{array}{l}
v_{m} \rightarrow v \quad \text { in } L^{\infty}(0, T ; V) \text { weak* } \\
\left(t v_{m}\right)^{\prime} \rightarrow(t v)^{\prime} \quad \text { in } L^{2}(0, T ; V) \text { weak. }
\end{array}\right.
$$

Using a compactness lemma ([19], Lions, p.57) applied to (3.25), we can extract from the sequence $\left\{v_{m}\right\}$ a subsequence, still denoted by $\left\{v_{m}\right\}$, such that

$$
t v_{m} \rightarrow t v \quad \text { strongly in } L^{2}\left(Q_{T}\right) .
$$

By the Riesz-Fischer theorem, we can extract from $\left\{v_{m}\right\}$ a subsequence, still denoted by $\left\{v_{m}\right\}$, such that

$$
v_{m}(x, t) \rightarrow v(x, t) \quad \text { a.e. }(x, t) \text { in } Q_{T} .
$$

Because $f$ is continuous, then

$$
f\left(v_{m}(x, t)+\varphi(x, t)\right) \rightarrow f(v(x, t)+\varphi(x, t)) \quad \text { a.e. }(x, t) \text { in } Q_{T} .
$$

On the other hand, by $\left(\mathrm{H}_{4}\right)$, it follows from (3.21) that

$$
\left|f\left(v_{m}(x, t)+\varphi(x, t)\right)\right| \leq \sup _{|z| \leq \bar{C}_{T}}|f(z)| \leq C_{T},
$$

where $C_{T}$ is a constant independent of $m$. 
Using the dominated convergence theorem, (3.28), and (3.29) yield

$$
f\left(v_{m}+\varphi\right) \rightarrow f(v+\varphi) \quad \text { strongly in } L^{2}\left(Q_{T}\right)
$$

Passing to the limit in (3.7) by (3.8), (3.25), (3.30), we have

$$
\left\{\begin{array}{l}
\frac{d}{d t}[\langle v(t), w\rangle+\alpha a(v(t), w)]+\mu a(v(t), w)+\langle f(v(t)+\varphi(t)), w\rangle \\
\quad=\left\langle f_{2}(t), w\right\rangle \quad \text { for all } w \in V, \text { a.e. } t \in(0, T) \\
v(0)=\tilde{v}_{0}
\end{array}\right.
$$

Step 4. Uniqueness of the solution.

First, we shall need the following lemma.

Lemma 3.2 Let $v$ be the weak solution of the following problem:

$$
\left\{\begin{array}{l}
v_{t}-\left(\mu+\alpha \frac{\partial}{\partial t}\right)\left(\frac{\partial^{2} v}{\partial x^{2}}+\frac{1}{x} \frac{\partial v}{\partial x}\right)=\tilde{f}(x, t), \quad 1<x<R, 0<t<T, \\
v_{x}(1, t)-h_{1} v(1, t)=v(R, t)=0 \\
v(x, 0)=\tilde{v}_{0}(x), \\
v \in L^{\infty}(0, T ; V), \quad t v_{t} \in L^{2}(0, T ; V) .
\end{array}\right.
$$

Then

$$
\begin{gathered}
\|v(t)\|_{0}^{2}+\alpha a(v(t), v(t))+2 \mu \int_{0}^{t} a(v(s), v(s)) d s \\
\geq\left\|\tilde{v}_{0}\right\|_{0}^{2}+\alpha a\left(\tilde{v}_{0}, \tilde{v}_{0}\right)+2 \int_{0}^{t}\langle\tilde{f}(s), v(s)\rangle d s .
\end{gathered}
$$

Furthermore, if $\tilde{v}_{0}=0$ then the equality in (3.33) follows.

Lemma 3.2 is a slight improvement of a lemma used in [14] (see also Lions' book [19]). Now, we will prove the uniqueness of the solution.

Let $v_{1}$ and $v_{2}$ be two weak solutions of (3.1). Then $v=v_{1}-v_{2}$ is a weak solution of Problem (3.32) with the right-hand side function replaced by $\tilde{f}(x, t)=-f\left(v_{1}+\varphi\right)+f\left(v_{2}+\varphi\right)$ and $\tilde{v}_{0}=0$. Using Lemma 3.2, we get

$$
\begin{gathered}
\|v(t)\|_{0}^{2}+\alpha a(v(t), v(t))+2 \mu \int_{0}^{t} a(v(s), v(s)) d s \\
=-2 \int_{0}^{t}\left\langle f\left(v_{1}+\varphi\right)-f\left(v_{2}+\varphi\right), v(s)\right\rangle d s .
\end{gathered}
$$

By $\left(\mathrm{H}_{4}\right)$, we obtain

$$
\int_{0}^{t}\left\langle f\left(v_{1}+\varphi\right)-f\left(v_{2}+\varphi\right), v(s)\right\rangle d s \geq-\delta \int_{0}^{t}\|v(s)\|_{0}^{2} d s .
$$

It follows from (3.34), (3.35) that

$$
\begin{aligned}
\sigma(t) & \equiv\|v(t)\|_{0}^{2}+\alpha\left\|v_{x}(t)\right\|_{0}^{2}+2 \mu \int_{0}^{t}\left\|v_{x}(s)\right\|_{0}^{2} d s \\
& \leq 2 \delta \int_{0}^{t}\|v(s)\|_{0}^{2} d s \leq 2 \delta \int_{0}^{t} \sigma(s) d s .
\end{aligned}
$$


By Gronwall's lemma $v=v_{1}-v_{2}=0$.

Assume now that $\left(\mathrm{H}_{3}\right)$ is replaced by $f_{1} \in L^{2}\left(Q_{T}\right)$, then we only show that $\left\{v_{m}^{\prime}\right\}$ is bounded in $L^{2}(0, T ; V)$.

Indeed, multiplying the $j$ th equation of (3.7) by $c_{m j}^{\prime}(t)$ and summing up with respect to $j$, afterward, integrating with respect to the time variable from 0 to $t$, we get after some rearrangements

$$
\begin{aligned}
& 2 \int_{0}^{t}\left(\left\|v_{m}^{\prime}(s)\right\|_{0}^{2}+\alpha a\left(v_{m}^{\prime}(s), v_{m}^{\prime}(s)\right)\right) d s+\mu a\left(v_{m}(t), v_{m}(t)\right) \\
& \quad=\mu a\left(v_{0 m}, v_{0 m}\right)+2 \int_{0}^{t}\left\langle f_{2}(s), v_{m}^{\prime}(s)\right\rangle d s-2 \int_{0}^{t}\left\langle f\left(v_{m}(s)+\varphi(s)\right), v_{m}^{\prime}(s)\right\rangle d s
\end{aligned}
$$

By the same estimates as above, we obtain

$$
\left\{\begin{array}{l}
\mu a\left(v_{0 m}, v_{0 m}\right) \leq \frac{\mu}{\alpha} C_{0} \\
2 \int_{0}^{t}\left\langle f_{2}(s), v_{m}^{\prime}(s)\right\rangle d s \leq 2\left\|f_{2}\right\|_{L^{2}\left(Q_{T}\right)}^{2}+\frac{1}{2} \int_{0}^{t}\left\|v_{m}^{\prime}(s)\right\|_{0}^{2} d s \\
-2 \int_{0}^{t}\left\langle f\left(v_{m}(s)+\varphi(s)\right), v_{m}^{\prime}(s)\right\rangle d s \leq T\left(R^{2}-1\right) \sup _{|z| \leq \bar{C}_{T}} f^{2}(z)+\int_{0}^{t}\left\|v_{m}^{\prime}(s)\right\|_{0}^{2} d s .
\end{array}\right.
$$

This implies

$$
\begin{gathered}
\int_{0}^{t}\left(\left\|v_{m}^{\prime}(s)\right\|_{0}^{2}+\alpha a\left(v_{m}^{\prime}(s), v_{m}^{\prime}(s)\right)\right) d s+\mu a\left(v_{m}(t), v_{m}(t)\right) \\
\quad \leq \frac{\mu}{\alpha} C_{0}+2\left\|f_{2}\right\|_{L^{2}\left(Q_{T}\right)}^{2}+T\left(R^{2}-1\right) \sup _{|z| \leq \bar{C}_{T}} f^{2}(z) \leq C_{T} .
\end{gathered}
$$

Then the sequence $\left\{v_{m}^{\prime}\right\}$ is bounded in $L^{2}(0, T ; V)$.

Applying a similar argument used in the proof of Theorem 3.1, the limit $v$ of the sequence $\left\{v_{m}\right\}$ in suitable function spaces, is a unique weak solution of Problem (3.1) satisfying (3.5).

Therefore, Theorem 3.1 is proved.

\section{Asymptotic behavior of the solution as $\boldsymbol{t} \rightarrow+\infty$}

In this part, let $T>0,\left(\mathrm{H}_{1}\right)-\left(\mathrm{H}_{4}\right)$ hold. Then there exists a unique solution $u=v+\varphi$ of Problem (1.1)-(1.3) such that

$$
u-\varphi=v \in L^{\infty}(0, T ; V) \text { and } \quad t\left(u_{t}-\varphi_{t}\right)=t v_{t} \in L^{2}(0, T ; V) .
$$

We shall study asymptotic behavior of the solution $u(t)$ as $t \rightarrow+\infty$.

We make the following supplementary assumptions on the functions $f_{1}(x, t), g_{1}(t), g_{R}(t)$ :

$\left(\mathrm{H}_{2}^{\prime}\right) g_{1}, g_{R} \in W^{1,1}\left(\mathbb{R}_{+}\right), \tilde{u}_{0 x}(1)-h_{1} \tilde{u}_{0}(1)=g_{1}(0), \tilde{u}_{0}(R)=g_{R}(0)$, there exist the positive constants $\bar{C}_{1}, \bar{C}_{R}, \bar{\gamma}_{1}, \bar{\gamma}_{R}$, such that

$$
\left|g_{i}(t)\right|+\left|g_{i}^{\prime}(t)\right| \leq \bar{C}_{i} e^{-\bar{\gamma}_{i} t}, \quad \forall t \geq 0, i \in\{1, R\}
$$

$\left(\mathrm{H}_{3}^{\prime}\right) f_{1} \in L^{\infty}\left(0, \infty ; L^{2}\right)$, there exist the positive constants $C_{1}, \gamma_{1}$ and the function $f_{1 \infty} \in L^{2}$, such that

$$
\left\|f_{1}(t)-f_{1 \infty}\right\|_{0} \leq C_{1} e^{-\gamma_{1} t} \quad \forall t \geq 0
$$


$\left(\mathrm{H}_{4}^{\prime}\right) f \in C^{0}(\mathbb{R} ; \mathbb{R})$ satisfies the condition that there exists a positive constant $\delta$, with $0<\delta<$ $\frac{2 \mu}{R(R-1)^{2}}$, such that

$$
(y-z)(f(y)-f(z)) \geq-\delta|y-z|^{2} \quad \text { for all } y, z \in \mathbb{R}
$$

First, we consider the following stationary problem:

$$
\left\{\begin{array}{l}
-\mu\left(\frac{\partial^{2} u}{\partial x^{2}}+\frac{1}{x} \frac{\partial u}{\partial x}\right)+f(u)=f_{1 \infty}(x), \quad 1<x<R \\
u_{x}(1, t)-h_{1} u(1, t)=u(R, t)=0
\end{array}\right.
$$

The weak solution of problem (4.1) is obtained from the following variational problem: Find $u_{\infty} \in V$ such that

$$
\mu a\left(u_{\infty}, w\right)+\left\langle f\left(u_{\infty}\right), w\right\rangle=\left\langle f_{1 \infty}, w\right\rangle
$$

for all $w \in V$, where $a(\cdot, \cdot)$ is the symmetric bilinear form on $V \times V$ defined by (2.6).

We then have the following theorem.

Theorem 4.1 Let $\left(\mathrm{H}_{3}^{\prime}\right),\left(\mathrm{H}_{4}^{\prime}\right)$ hold. Then there exists a unique solution $u_{\infty}$ of the variational problem (4.2) such that $u_{\infty} \in V$.

Proof Consider the basis $\left\{w_{j}\right\}$ for $V$ as in Lemma 2.4. Put

$$
y_{m}=\sum_{j=1}^{m} d_{m j} w_{j}
$$

where $d_{m j}$ satisfy the following nonlinear equation system:

$$
\mu a\left(y_{m}, w_{j}\right)+\left\langle f\left(y_{m}\right), w_{j}\right\rangle=\left\langle f_{1 \infty}, w_{j}\right\rangle, \quad 1 \leq j \leq m
$$

By Brouwer's lemma (see Lions [19], Lemma 4.3, p.53), it follows from the hypotheses $\left(\mathrm{H}_{3}^{\prime}\right),\left(\mathrm{H}_{4}^{\prime}\right)$ that system (4.3), (4.4) has a solution $y_{m}$.

Multiplying the $j$ th equation of system (4.4) by $d_{m j}$, then summing up with respect to $j$, we have

$$
\mu a\left(y_{m}, y_{m}\right)+\left\langle f\left(y_{m}\right), y_{m}\right\rangle=\left\langle f_{1 \infty}, y_{m}\right\rangle
$$

By using $\left(\mathrm{H}_{4}\right)$, we obtain

$$
\begin{aligned}
\left\langle f\left(y_{m}\right), y_{m}\right\rangle & =\int_{1}^{R} x\left(f\left(y_{m}(x)\right)-f(0)\right) y_{m}(x) d x+\int_{1}^{R} x f(0) y_{m}(x) d x \\
& \geq-\delta \int_{1}^{R} x y_{m}^{2}(x) d x+\int_{1}^{R} x f(0) y_{m}(x) d x \\
& \geq-\delta\left\|y_{m}\right\|_{0}^{2}-\varepsilon_{1}\left\|y_{m}\right\|_{0}^{2}-\frac{1}{4 \varepsilon_{1}} \int_{1}^{R} x f^{2}(0) d x \\
& =-\left(\delta+\varepsilon_{1}\right)\left\|y_{m}\right\|_{0}^{2}-\frac{1}{8 \varepsilon_{1}}\left(R^{2}-1\right) f^{2}(0) .
\end{aligned}
$$


By using the inequalities (2.5)(iii), (4.6), we obtain from (4.5)

$$
\begin{aligned}
\mu\left\|y_{m x}\right\|_{0}^{2} & \leq \mu a\left(y_{m}, y_{m}\right) \leq\left(\delta+\varepsilon_{1}\right)\left\|y_{m}\right\|_{0}^{2}+\frac{1}{8 \varepsilon_{1}}\left(R^{2}-1\right) f^{2}(0)+\left\|f_{1 \infty}\right\|_{0}\left\|y_{m}\right\|_{0} \\
& \leq\left(\delta+\varepsilon_{1}\right)\left\|y_{m}\right\|_{0}^{2}+\frac{1}{8 \varepsilon_{1}}\left(R^{2}-1\right) f^{2}(0)+\frac{1}{4 \varepsilon_{1}}\left\|f_{1 \infty}\right\|_{0}^{2}+\varepsilon_{1}\left\|y_{m}\right\|_{0}^{2} \\
& \leq\left(\delta+2 \varepsilon_{1}\right) \frac{R}{2}(R-1)^{2}\left\|y_{m x}\right\|_{0}^{2}+\frac{1}{8 \varepsilon_{1}}\left(\left(R^{2}-1\right) f^{2}(0)+2\left\|f_{1 \infty}\right\|_{0}^{2}\right) .
\end{aligned}
$$

By $0<\delta<\frac{2 \mu}{R(R-1)^{2}}$, choose $\varepsilon_{1}>0$ such that $0<\delta+2 \varepsilon_{1}<\frac{2 \mu}{R(R-1)^{2}}$.

Hence, we deduce from (4.7) that

$$
\left\|y_{m x}\right\|_{0} \leq \tilde{C}
$$

$\tilde{C}$ is a constant independent of $m$.

By means of (4.8) and Lemma 2.3, the sequence $\left\{y_{m}\right\}$ has a subsequence still denoted by $\left\{y_{m}\right\}$ such that

$$
\begin{cases}y_{m} \rightarrow u_{\infty} & \text { in } V \text { weakly, } \\ y_{m} \rightarrow u_{\infty} & \text { in } C^{0}([1, R]) \text { strongly. }\end{cases}
$$

On the other hand, by $(4.9)_{2}$ and the continuity of $f$, we have

$$
f\left(y_{m}\right) \rightarrow f\left(u_{\infty}\right) \quad \text { in } C^{0}([1, R]) \text { strongly. }
$$

Passing to the limit in equation (4.4), we find without difficulty from (4.9), (4.10) that $u_{\infty}$ satisfies the equation

$$
\mu a\left(u_{\infty}, w_{j}\right)+\left\langle f\left(u_{\infty}\right), w_{j}\right\rangle=\left\langle f_{1 \infty}, w_{j}\right\rangle
$$

Equation (4.11) holds for every $j=1,2, \ldots$, i.e., (4.2) holds.

The solution of Problem (4.2) is unique, which can be showed by the same arguments as in the proof of Theorem 3.1.

This completes the proof of Theorem 4.1.

Now we consider asymptotic behavior of the solution $u(t)$ as $t \rightarrow+\infty$.

We then have the following theorem.

Theorem 4.2 Let $\left(\mathrm{H}_{1}\right),\left(\mathrm{H}_{2}^{\prime}\right)-\left(\mathrm{H}_{4}^{\prime}\right)$ hold. Let $f$ satisfy the following condition, in addition:

$$
\left(\mathrm{H}_{4}^{\prime \prime}\right) \quad \forall M>0, \exists k_{M}>0: \quad|f(y)-f(z)| \leq k_{M}|y-z|, \quad \forall y, z \in[-M, M] .
$$

Then we have

$$
\left\|u(t)-u_{\infty}\right\|_{1} \leq \bar{C} e^{-\gamma t}, \quad \forall t \geq 0
$$

where $\gamma>0, \bar{C}>0$ are constants independent of $t$. 
Proof Put $Z_{m}(t)=v_{m}(t)-y_{m}$. Let us subtract (4.4) from (3.7) $)_{1}$ to obtain

$$
\left\{\begin{array}{l}
\left\langle Z_{m}^{\prime}(t), w_{j}\right\rangle+\alpha a\left(Z_{m}^{\prime}(t), w_{j}\right)+\mu a\left(Z_{m}(t), w_{j}\right)+\left\langle f\left(v_{m}(t)+\varphi(t)\right)-f\left(y_{m}\right), w_{j}\right\rangle \\
\quad=\left\langle f_{2}(t)-f_{1 \infty}, w_{j}\right\rangle, \quad 1 \leq j \leq m, \\
Z_{m}(0)=v_{0 m}-y_{m} .
\end{array}\right.
$$

By multiplying $(4.14)_{1}$ by $c_{m j}(t)-d_{m j}$ and summing up in $j$, we obtain

$$
\begin{aligned}
\frac{1}{2} \frac{d}{d t} & {\left[\left\|Z_{m}(t)\right\|_{0}^{2}+\alpha a\left(Z_{m}(t), Z_{m}(t)\right)\right]+\mu a\left(Z_{m}(t), Z_{m}(t)\right) } \\
& \quad+\left\langle f\left(v_{m}(t)+\varphi(t)\right)-f\left(y_{m}+\varphi(t)\right), Z_{m}(t)\right\rangle+\left\langle f\left(y_{m}+\varphi(t)\right)-f\left(y_{m}\right), Z_{m}(t)\right\rangle \\
= & \left\langle f_{2}(t)-f_{1}(t), Z_{m}(t)\right\rangle+\left\langle f_{1}(t)-f_{1 \infty}, Z_{m}(t)\right\rangle .
\end{aligned}
$$

By the assumptions $\left(\mathrm{H}_{1}\right)-\left(\mathrm{H}_{4}\right),\left(\mathrm{H}_{2}^{\prime}\right)-\left(\mathrm{H}_{4}^{\prime}\right),\left(\mathrm{H}_{4}^{\prime \prime}\right)$ and using the inequality (2.5)(iii), and with $\varepsilon_{2}>0$, we estimate without difficulty the following terms in (4.15):

(i) Estimate $\left\langle f\left(v_{m}(t)+\varphi(t)\right)-f\left(y_{m}+\varphi(t)\right), Z_{m}(t)\right\rangle$ :

$$
\begin{aligned}
\left\langle f\left(v_{m}(t)+\varphi(t)\right)-f\left(y_{m}+\varphi(t)\right), Z_{m}(t)\right\rangle & \geq-\delta\left\|Z_{m}(t)\right\|_{0}^{2} \geq-\delta \frac{R}{2}(R-1)^{2}\left\|Z_{m x}(t)\right\|_{0}^{2} \\
& \geq-\delta \frac{R}{2}(R-1)^{2} a\left(Z_{m}(t), Z_{m}(t)\right) .
\end{aligned}
$$

(ii) Estimate $\left\langle f\left(y_{m}+\varphi(t)\right)-f\left(y_{m}\right), Z_{m}(t)\right\rangle$.

Note that from the inequalities

$$
\begin{aligned}
& |\varphi(x, t)| \leq\left[R+h_{1}(R-1)\right]\left[\left|g_{1}(t)\right|+\left|g_{R}(t)\right|\right] \leq\left[R+h_{1}(R-1)\right]\left(\bar{C}_{1}+\bar{C}_{R}\right), \\
& \left\|y_{m}\right\|_{C^{0}(\bar{\Omega})} \leq \sqrt{R-1}\left\|y_{m x}\right\| \leq \sqrt{R-1} \tilde{C} \\
& \left\|y_{m}+\varphi\right\|_{C^{0}(\bar{\Omega})} \leq \sqrt{R-1} \tilde{C}+\left[R+h_{1}(R-1)\right]\left(\bar{C}_{1}+\bar{C}_{R}\right)=M
\end{aligned}
$$

and $\left(\mathrm{H}_{4}^{\prime \prime}\right)$, we deduce that

$$
\left|f\left(y_{m}+\varphi(t)\right)-f\left(y_{m}\right)\right| \leq k_{M}|\varphi(x, t)| \leq k_{M}\left(\left|g_{1}(t)\right|+\left|g_{R}(t)\right|\right) \leq k_{M} \Psi(t),
$$

where

$$
\Psi(t)=\bar{C}_{1} e^{-\bar{\gamma}_{1} t}+\bar{C}_{R} e^{-\bar{\gamma}_{R} t} .
$$

Hence

$$
\left\|f\left(y_{m}+\varphi(t)\right)-f\left(y_{m}\right)\right\|_{0}^{2}=\int_{1}^{R} x\left|f\left(y_{m}+\varphi(t)\right)-f\left(y_{m}\right)\right|^{2} d x \leq \frac{1}{2}\left(R^{2}-1\right) k_{M}^{2} \Psi^{2}(t) .
$$

Thus

$$
\begin{aligned}
\left\langle f\left(y_{m}+\varphi(t)\right)-f\left(y_{m}\right), Z_{m}(t)\right\rangle \leq & \frac{1}{2 \varepsilon_{2}}\left\|f\left(y_{m}+\varphi(t)\right)-f\left(y_{m}\right)\right\|_{0}^{2}+\frac{\varepsilon_{2}}{2}\left\|Z_{m}(t)\right\|_{0}^{2} \\
\leq & \frac{1}{2 \varepsilon_{2}} \frac{1}{2}\left(R^{2}-1\right) k_{M}^{2} \Psi^{2}(t) \\
& +\frac{\varepsilon_{2}}{2} \frac{R}{2}(R-1)^{2} a\left(Z_{m}(t), Z_{m}(t)\right) .
\end{aligned}
$$


(iii) Estimate $\left\langle f_{2}(t)-f_{1}(t), Z_{m}(t)\right\rangle$.

We have

$$
\begin{aligned}
& f_{2}(x, t)-f_{1}(x, t)=-\frac{1}{1+(R-1) h_{1}}\left[(x-R) g_{1}^{\prime}(t)+\left[h_{1}(x-1)+1\right] g_{R}^{\prime}(t)\right] \\
&+\frac{1}{1+(R-1) h_{1}}\left[\frac{\mu}{x}\left(g_{1}(t)+h_{1} g_{R}(t)\right)+\frac{\alpha}{x}\left(g_{1}^{\prime}(t)+h_{1} g_{R}^{\prime}(t)\right)\right], \\
&|\varphi(x, t)| \leq\left[R+h_{1}(R-1)\right]\left[\left|g_{1}(t)\right|+\left|g_{R}(t)\right|\right], \\
&\left|\varphi_{t}(x, t)\right| \leq\left[R+h_{1}(R-1)\right]\left[\left|g_{1}^{\prime}(t)\right|+\left|g_{R}^{\prime}(t)\right|\right] .
\end{aligned}
$$

Hence

$$
\begin{aligned}
\left|f_{2}(x, t)-f_{1}(x, t)\right| \leq & {\left[R+h_{1}(R-1)\right]\left(\left|g_{1}^{\prime}(t)\right|+\left|g_{R}^{\prime}(t)\right|\right) } \\
& +\frac{1}{1+(R-1) h_{1}}(\alpha+\mu)\left[\left|g_{1}(t)\right|+\left|g_{1}^{\prime}(t)\right|+\left|g_{R}(t)\right|+\left|g_{R}^{\prime}(t)\right|\right] \\
\leq & {\left[R+h_{1}(R-1)\right] \Psi(t)+\frac{1}{1+(R-1) h_{1}}(\alpha+\mu) \Psi(t) } \\
= & \left(R+h_{1}(R-1)+\frac{\alpha+\mu}{1+(R-1) h_{1}}\right) \Psi(t) \equiv \bar{D}_{R} \Psi(t) .
\end{aligned}
$$

It follows that

$$
\left\|f_{2}(t)-f_{1}(t)\right\|_{0}^{2}=\int_{1}^{R} x\left|f_{2}(x, t)-f_{1}(x, t)\right|^{2} d x \leq \frac{1}{2}\left(R^{2}-1\right) \bar{D}_{R}^{2} \Psi^{2}(t) .
$$

Thus

$$
\begin{aligned}
\left\langle f_{2}(t)-f_{1}(t), Z_{m}(t)\right\rangle & \leq \frac{1}{2 \varepsilon_{2}}\left\|f_{2}(t)-f_{1}(t)\right\|_{0}^{2}+\frac{\varepsilon_{2}}{2}\left\|Z_{m}(t)\right\|_{0}^{2} \\
& \leq \frac{1}{2 \varepsilon_{2}} \frac{1}{2}\left(R^{2}-1\right) \bar{D}_{R}^{2} \Psi^{2}(t)+\frac{\varepsilon_{2}}{2} \frac{R}{2}(R-1)^{2} a\left(Z_{m}(t), Z_{m}(t)\right) .
\end{aligned}
$$

(iv) Estimate $\left\langle f_{1}(t)-f_{1 \infty}, Z_{m}(t)\right\rangle$.

$$
\begin{aligned}
\left\langle f_{1}(t)-f_{1 \infty}, Z_{m}(t)\right\rangle & \leq \frac{1}{2 \varepsilon_{2}}\left\|f_{1}(t)-f_{1 \infty}\right\|_{0}^{2}+\frac{\varepsilon_{2}}{2}\left\|Z_{m}(t)\right\|_{0}^{2} \\
& \leq \frac{1}{2 \varepsilon_{2}} C_{1}^{2} e^{-2 \gamma_{1} t}+\frac{\varepsilon_{2}}{2} \frac{R}{2}(R-1)^{2} a\left(Z_{m}(t), Z_{m}(t)\right) .
\end{aligned}
$$

It follows from (4.15), (4.16), (4.21), (4.25), and (4.26) that

$$
\begin{aligned}
& \frac{1}{2} \frac{d}{d t}\left[\left\|Z_{m}(t)\right\|_{0}^{2}+\alpha a\left(Z_{m}(t), Z_{m}(t)\right)\right]+\left[\mu-\left(\delta+\frac{3 \varepsilon_{2}}{2}\right) \frac{R}{2}(R-1)^{2}\right] a\left(Z_{m}(t), Z_{m}(t)\right) \\
& \quad \leq \frac{1}{4 \varepsilon_{2}}\left[2 C_{1}^{2} e^{-2 \gamma_{1} t}+\left(R^{2}-1\right)\left(k_{M}^{2}+\bar{D}_{R}^{2}\right) \Psi^{2}(t)\right]=\tilde{\psi}(t)
\end{aligned}
$$

By $0<\delta<\frac{2 \mu}{R(R-1)^{2}}$, choose $\varepsilon_{2}>0$ such that $\tilde{\gamma}=\mu-\left(\delta+\frac{3 \varepsilon_{2}}{2}\right) \frac{R}{2}(R-1)^{2}>0$. 
Put $\bar{\gamma}_{0}=\min \left\{\gamma_{1}, \bar{\gamma}_{1}, \bar{\gamma}_{R}\right\}$, we have $\tilde{\psi}(t) \leq \bar{C}_{0} e^{-2 \bar{\gamma}_{0} t}$ for all $t \geq 0$ and

$$
\frac{d}{d t}\left[\left\|Z_{m}(t)\right\|_{0}^{2}+\alpha a\left(Z_{m}(t), Z_{m}(t)\right)\right]+2 \tilde{\gamma} a\left(Z_{m}(t), Z_{m}(t)\right) \leq 2 \tilde{\psi}(t)=2 \bar{C}_{0} e^{-2 \bar{\gamma} 0 t}
$$

By

$$
\begin{aligned}
a\left(Z_{m}(t), Z_{m}(t)\right) & =\frac{1}{2} a\left(Z_{m}(t), Z_{m}(t)\right)+\frac{1}{2} a\left(Z_{m}(t), Z_{m}(t)\right) \\
& \geq \frac{1}{2} a\left(Z_{m}(t), Z_{m}(t)\right)+\frac{1}{2}\left\|Z_{m x}(t)\right\|_{0}^{2} \\
& \geq \frac{1}{2} \frac{1}{\alpha} \alpha a\left(Z_{m}(t), Z_{m}(t)\right)+\frac{1}{2} \frac{2}{R(R-1)^{2}}\left\|Z_{m}(t)\right\|_{0}^{2} \\
& \geq \beta_{1}\left(\left\|Z_{m}(t)\right\|_{0}^{2}+\alpha a\left(Z_{m}(t), Z_{m}(t)\right)\right),
\end{aligned}
$$

where $\beta_{1}=\frac{1}{2} \min \left\{\frac{1}{\alpha}, \frac{2}{R(R-1)^{2}}\right\}$.

It follows from (4.28), (4.29) that

$$
\begin{aligned}
& \frac{d}{d t}\left[\left\|Z_{m}(t)\right\|_{0}^{2}+\alpha a\left(Z_{m}(t), Z_{m}(t)\right)\right] \\
& \quad+2 \tilde{\gamma} \beta_{1}\left(\left\|Z_{m}(t)\right\|_{0}^{2}+\alpha a\left(Z_{m}(t), Z_{m}(t)\right)\right) \leq 2 \bar{C}_{0} e^{-2 \bar{\gamma}_{0} t} .
\end{aligned}
$$

Choose $\gamma>0$ such that $\gamma<\min \left\{\bar{\gamma}_{0}, 2 \tilde{\gamma} \beta_{1}\right\}$, then we have from (4.30)

$$
\begin{aligned}
& \frac{d}{d t}\left[\left\|Z_{m}(t)\right\|_{0}^{2}+\alpha a\left(Z_{m}(t), Z_{m}(t)\right)\right] \\
& \quad+2 \gamma\left[\left\|Z_{m}(t)\right\|_{0}^{2}+\alpha a\left(Z_{m}(t), Z_{m}(t)\right)\right] \leq 2 \bar{C}_{0} e^{-2 \bar{\gamma}_{0} t} .
\end{aligned}
$$

Hence, we obtain from (4.31)

$$
\begin{aligned}
& \left\|Z_{m}(t)\right\|_{0}^{2}+\alpha a\left(Z_{m}(t), Z_{m}(t)\right) \\
& \quad \leq\left[\left\|Z_{m}(0)\right\|_{0}^{2}+\alpha a\left(Z_{m}(0), Z_{m}(0)\right)+\frac{\bar{C}_{0}}{\bar{\gamma}_{0}-\gamma}\right] e^{-2 \gamma t} .
\end{aligned}
$$

Letting $m \rightarrow+\infty$ in (4.32) we obtain

$$
\begin{aligned}
& \left\|v(t)-u_{\infty}\right\|_{0}^{2}+\alpha a\left(v(t)-u_{\infty}, v(t)-u_{\infty}\right) \\
& \quad \leq \liminf _{m \rightarrow+\infty}\left[\left\|v_{m}(t)-y_{m}\right\|_{0}^{2}+\alpha a\left(v_{m}(t)-y_{m}, v_{m}(t)-y_{m}\right)\right] \\
& \quad \leq\left(\left\|\tilde{v}_{0}-u_{\infty}\right\|_{0}^{2}+\alpha a\left(\tilde{v}_{0}-u_{\infty}, \tilde{v}_{0}-u_{\infty}\right)+\frac{\bar{C}_{0}}{\bar{\gamma}_{0}-\gamma}\right) e^{-2 \gamma t} \quad \text { for all } t \geq 0,
\end{aligned}
$$

or

$$
\left\|v(t)-u_{\infty}\right\|_{1} \leq \tilde{D}_{1} e^{-\gamma t} \quad \text { for all } t \geq 0
$$

where

$$
\tilde{D}_{1}=\sqrt{\frac{1}{\min (1, \alpha)}\left(\left\|\tilde{v}_{0}-u_{\infty}\right\|_{0}^{2}+\alpha a\left(\tilde{v}_{0}-u_{\infty}, \tilde{v}_{0}-u_{\infty}\right)+\frac{\bar{C}_{0}}{\bar{\gamma}_{0}-\gamma}\right)} .
$$


Note that

$$
\begin{aligned}
|\varphi(x, t)| & \leq\left[R+h_{1}(R-1)\right]\left[\left|g_{1}(t)\right|+\left|g_{R}(t)\right|\right] \leq\left[R+h_{1}(R-1)\right]\left(\bar{C}_{1} e^{-\bar{\gamma}_{1} t}+\bar{C}_{R} e^{-\bar{\gamma}_{R} t}\right) \\
& =\left[R+h_{1}(R-1)\right] \Psi(t) ; \\
\left|\varphi_{x}(x, t)\right| & =\frac{g_{1}(t)+h_{1} g_{R}(t)}{1+(R-1) h_{1}} \leq \frac{1+h_{1}}{1+(R-1) h_{1}}\left[\left|g_{1}(t)\right|+\left|g_{R}(t)\right|\right] \\
& \leq \frac{1+h_{1}}{1+(R-1) h_{1}}\left(\bar{C}_{1} e^{-\bar{\gamma}_{1} t}+\bar{C}_{R} e^{-\bar{\gamma}_{R} t}\right) \leq \frac{1+h_{1}}{1+(R-1) h_{1}} \Psi(t) ; \\
\|\varphi(t)\|_{1}^{2} & =\|\varphi(t)\|_{0}^{2}+\left\|\varphi_{x}(t)\right\|_{0}^{2} \\
& \leq \frac{1}{2}\left(R^{2}-1\right)\left[\left[R+h_{1}(R-1)\right]^{2}+\left(\frac{1+h_{1}}{1+(R-1) h_{1}}\right)^{2}\right] \Psi^{2}(t) \leq \tilde{D}_{2}^{2} e^{-2 \gamma t} .
\end{aligned}
$$

It follows from (4.34), (4.36) that

$$
\begin{aligned}
\left\|u(t)-u_{\infty}\right\|_{1} & =\left\|v(t)+\varphi(t)-u_{\infty}\right\|_{1} \leq\left\|v(t)-u_{\infty}\right\|_{1}+\|\varphi(t)\|_{1} \leq \tilde{D}_{1} e^{-\gamma t}+\|\varphi(t)\|_{1} \\
& \leq\left(\tilde{D}_{1}+\tilde{D}_{2}\right) e^{-\gamma t} \quad \text { for all } t \geq 0 .
\end{aligned}
$$

This completes the proof of Theorem 4.2.

\section{The existence and uniqueness of a weak solution with respect to}

\section{$(N+1)$-points condition in time}

In this section, we shall consider Problem (1.1), (1.2), (1.3a) with $\mu>0, \alpha>0, R>1, h_{1} \geq 0$ being given constants and $T_{i}, \eta_{i}, i=1, \ldots, N$, are given constants satisfying (1.4).

We make the following assumptions:

$\left(\overline{\mathrm{H}}_{2}\right) g_{1}, g_{R} \in W^{1,1}(0, T), g_{1}, g_{R}$ satisfying the $(N+1)$-points condition in $t$, i.e.,

$$
g_{1}(0)=\sum_{i=1}^{N} \eta_{i} g_{1}\left(T_{i}\right), \quad g_{R}(0)=\sum_{i=1}^{N} \eta_{i} g_{R}\left(T_{i}\right)
$$

$\left(\overline{\mathrm{H}}_{3}\right) f_{1}, f_{1}^{\prime} \in L^{2}\left(Q_{T}\right), f_{1}$ satisfying the $(N+1)$-points condition in time, i.e., $f_{1}(x, 0)=$ $\sum_{i=1}^{N} \eta_{i} f_{1}\left(x, T_{i}\right)$.

Remark 5.1 An example of the functions $g_{1}, g_{R}$ satisfying $\left(\overline{\mathrm{H}}_{2}\right)$ are

$$
g_{k}(t)=\beta_{k} e^{p t},
$$

where $p>0, \beta_{k}, k \in\{1, R\}$ are constants. It is obvious that $\left(\overline{\mathrm{H}}_{2}\right)$ holds, because

$$
g_{k}(t)=\sum_{i=1}^{N} \frac{1}{N} e^{-p T_{i}} \beta_{k} e^{p\left(t+T_{i}\right)}=\sum_{i=1}^{N} \frac{1}{N} e^{-p T_{i}} g_{k}\left(t+T_{i}\right)=\sum_{i=1}^{N} \eta_{i} g_{k}\left(t+T_{i}\right)
$$

with

$$
\eta_{i}=\frac{1}{N} e^{-p T_{i}}, \quad i=1, \ldots, N,
$$


and

$$
\begin{aligned}
& g_{k}(0)=\sum_{i=1}^{N} \eta_{i} g_{k}\left(T_{i}\right), \quad k \in\{1, R\}, \\
& \sum_{i=1}^{N}\left|\eta_{i}\right|=\sum_{i=1}^{N} \frac{1}{N} e^{-p T_{i}} \leq \sum_{i=1}^{N} \frac{1}{N}=1 .
\end{aligned}
$$

Similarly, by the transformation $v(x, t)=u(x, t)-\varphi(x, t)$, with

$$
\varphi(x, t)=\frac{(x-R) g_{1}(t)+\left[h_{1}(x-1)+1\right] g_{R}(t)}{1+(R-1) h_{1}}
$$

and by $\varphi(x, 0)=\sum_{i=1}^{N} \eta_{i} \varphi\left(0, T_{i}\right)$, Problem (1.1), (1.2), (1.3a) reduces to the following problem:

$$
\left\{\begin{array}{l}
v_{t}-\left(\mu+\alpha \frac{\partial}{\partial t}\right)\left(\frac{\partial^{2} v}{\partial x^{2}}+\frac{1}{x} \frac{\partial v}{\partial x}\right)+f(v+\varphi)=f_{2}(x, t), \quad 1<x<R, 0<t<T \\
v_{x}(1, t)-h_{1} v(1, t)=v(R, t)=0 \\
v(x, 0)=\sum_{i=1}^{N} \eta_{i} v\left(x, T_{i}\right)
\end{array}\right.
$$

where $f_{2}(x, t)$ is defined by $(3.2)_{1}$.

Remark 5.2 The weak formulation of Problem (5.1) can be given in the following manner: Find $v \in L^{\infty}(0, T ; V)$ with $v_{t} \in L^{2}(0, T ; V)$, such that $v$ satisfies the following variational equation:

$$
\left\{\begin{array}{l}
\int_{0}^{T}\left\langle v^{\prime}(t), w(t)\right\rangle d t+\alpha \int_{0}^{T} a\left(v^{\prime}(t), w(t)\right) d t+\mu \int_{0}^{T} a(v(t), w(t)) d t \\
\quad+\int_{0}^{T}\langle f(v(t)+\varphi(t)), w(t)\rangle d t \\
\quad=\int_{0}^{T}\left\langle f_{2}(t), w(t)\right\rangle d t \quad \text { for all } w \in L^{2}(0, T ; V), \\
v(0)=\sum_{i=1}^{N} \eta_{i} v\left(T_{i}\right),
\end{array}\right.
$$

where $a(\cdot, \cdot)$ is the symmetric bilinear form on $V \times V$ defined by (2.6).

Then we have the following theorem.

Theorem 5.1 Let $T>0$ and $\left(\overline{\mathrm{H}}_{2}\right),\left(\overline{\mathrm{H}}_{3}\right),\left(\mathrm{H}_{4}^{\prime}\right)$ hold. Then Problem (5.1) has a ' $(N+1)$-points condition in time' weak solution $v$ such that

$$
v \in L^{\infty}(0, T ; V) \quad \text { and } \quad v_{t} \in L^{2}(0, T ; V) .
$$

Furthermore, if $N=1$, then the solution is unique.

Proof The proof consists of several steps.

Step 1. The Faedo-Galerkin approximation (introduced by Lions [19]).

Consider the basis $\left\{w_{j}\right\}$ for $V$ as in Lemma 2.4. Let $W_{m}$ be the linear space generated by $w_{1}, w_{2}, \ldots, w_{m}$. We consider the following problem: 
Find a function $v_{m}(t)$ in the form (3.6) satisfying the nonlinear differential equation system $(3.7)_{1}$ and the $(N+1)$-points condition in time

$$
v_{m}(0)=\sum_{i=1}^{N} \eta_{i} v_{m}\left(T_{i}\right) .
$$

We consider the initial value problem given by (3.7), where $v_{0 m}$ is given in $W_{m}$.

It is clear that for each $m$, there exists a solution $v_{m}(t)$ in the form (3.6) which satisfies (3.7) almost everywhere on $0 \leq t \leq \tilde{T}_{m}$ for some $\tilde{T}_{m}, 0<\tilde{T}_{m} \leq T$. The following a priori estimates allow us to take $\tilde{T}_{m}=T$ for all $m$.

Step 2. A priori estimates.

Multiplying the $j$ th equation of $(3.7)_{1}$ by $c_{m j}(t)$ and summing up with respect to $j$, we get

$$
\begin{aligned}
& \frac{d}{d t}\left[\left\|v_{m}(t)\right\|_{0}^{2}+\alpha a\left(v_{m}(t), v_{m}(t)\right)\right] \\
& \quad+2 \mu a\left(v_{m}(t), v_{m}(t)\right)+2\left\langle f\left(v_{m}(t)+\varphi(t)\right), v_{m}(t)\right\rangle=2\left\langle f_{2}(t), v_{m}(t)\right\rangle .
\end{aligned}
$$

By the same estimates as in Section 3, and with $\varepsilon_{1}>0$, we obtain

$$
\begin{aligned}
& 2\left\langle f\left(v_{m}(t)+\varphi(t)\right), v_{m}(t)\right\rangle=2\left\langle f\left(v_{m}(t)+\varphi(t)\right)-f(\varphi(t)), v_{m}(t)\right\rangle+2\left\langle f(\varphi(t)), v_{m}(t)\right\rangle \\
& \geq-\left(2 \delta+\varepsilon_{1}\right) \frac{R}{2}(R-1)^{2} a\left(v_{m}(t), v_{m}(t)\right)-\frac{1}{\varepsilon_{1}}\|f(\varphi(t))\|_{0}^{2} ; \\
& 2\left\langle f_{2}(t), v_{m}(t)\right\rangle \leq \frac{1}{\varepsilon_{1}}\left\|f_{2}(t)\right\|_{0}^{2}+\varepsilon_{1}\left\|v_{m}(t)\right\|_{0}^{2} \leq \frac{1}{\varepsilon_{1}}\left\|f_{2}(t)\right\|_{0}^{2}+\varepsilon_{1} \frac{R}{2}(R-1)^{2}\left\|v_{m x}(t)\right\|_{0}^{2} \\
& \leq \frac{1}{\varepsilon_{1}}\left\|f_{2}(t)\right\|_{0}^{2}+\varepsilon_{1} \frac{R}{2}(R-1)^{2} a\left(v_{m}(t), v_{m}(t)\right) .
\end{aligned}
$$

Hence, it follows from (5.5)-(5.7) that

$$
\begin{aligned}
& \frac{d}{d t}\left[\left\|v_{m}(t)\right\|_{0}^{2}+\alpha a\left(v_{m}(t), v_{m}(t)\right)\right]+2\left[\mu-\left(\delta+\varepsilon_{1}\right) \frac{R}{2}(R-1)^{2}\right] a\left(v_{m}(t), v_{m}(t)\right) \\
& \quad \leq \frac{1}{\varepsilon_{1}}\left(\left\|f_{2}(t)\right\|_{0}^{2}+\|f(\varphi(t))\|_{0}^{2}\right) .
\end{aligned}
$$

By $0<\delta<\frac{2 \mu}{R(R-1)^{2}}$, choose $\varepsilon_{1}>0$ such that $\mu-\left(\delta+\varepsilon_{1}\right) \frac{R}{2}(R-1)^{2}>0$.

Similar to (4.29), we get

$$
a\left(v_{m}(t), v_{m}(t)\right) \geq \beta_{1}\left(\alpha a\left(v_{m}(t), v_{m}(t)\right)+\left\|v_{m}(t)\right\|_{0}^{2}\right),
$$

where $\beta_{1}=\frac{1}{2} \min \left\{\frac{1}{\alpha}, \frac{2}{R(R-1)^{2}}\right\}$.

It follows from (5.8), (5.9) that

$$
\frac{d}{d t}\left[\left\|v_{m}(t)\right\|_{0}^{2}+\alpha a\left(v_{m}(t), v_{m}(t)\right)\right]+2 \gamma\left(\left\|v_{m}(t)\right\|_{0}^{2}+\alpha a\left(v_{m}(t), v_{m}(t)\right)\right) \leq f_{*}(t),
$$

where $\gamma=\beta_{1}\left[\mu-\left(\delta+\varepsilon_{1}\right) \frac{R}{2}(R-1)^{2}\right], f_{*}(t)=\frac{1}{\varepsilon_{1}}\left(\left\|f_{2}(t)\right\|_{0}^{2}+\|f(\varphi(t))\|_{0}^{2}\right)$. 
Integrating (5.10), we have

$$
\begin{aligned}
\left\|v_{m}(t)\right\|_{0}^{2}+\alpha a\left(v_{m}(t), v_{m}(t)\right) & \leq\left[\left\|v_{0 m}\right\|_{0}^{2}+\alpha a\left(v_{0 m}, v_{0 m}\right)+\int_{0}^{t} e^{2 \gamma s} f_{*}(s) d s\right] e^{-2 \gamma t} \\
& \leq \rho^{2}+\left(\left\|v_{0 m}\right\|_{0}^{2}+\alpha a\left(v_{0 m}, v_{0 m}\right)-\rho^{2}\right) e^{-2 \gamma t}
\end{aligned}
$$

where $\rho^{2}=\sup _{0 \leq t \leq T} \rho_{1}(t)$, with

$$
\rho_{1}(t)= \begin{cases}\frac{1}{e^{2 \gamma t}-1} \int_{0}^{t} e^{2 \gamma s} f_{*}(s) d s, & 0<t \leq T \\ \frac{1}{2 \gamma} f_{*}(0), & t=0\end{cases}
$$

Therefore, if we choose $v_{0 m}$ such that $\left\|v_{0 m}\right\|_{0}^{2}+\alpha a\left(v_{0 m}, v_{0 m}\right) \leq \rho^{2}$, we obtain from (5.11) that

$$
\left\|v_{m}(t)\right\|_{0}^{2}+\alpha a\left(v_{m}(t), v_{m}(t)\right) \leq \rho^{2}, \quad \text { i.e., } \tilde{T}_{m}=T \text { for all } m
$$

hence

$$
\left\|v_{m}\left(T_{i}\right)\right\|_{0}^{2}+\alpha a\left(v_{m}\left(T_{i}\right), v_{m}\left(T_{i}\right)\right) \leq \rho^{2} \quad \text { for all } i=1,2, \ldots, N \text {, and for all } m
$$

In the space $W_{m}$ of linear combinations of the functions $w_{1}, w_{2}, \ldots, w_{m}$, we consider the norm $v_{0 m} \longmapsto\left\|v_{0 m}\right\|_{*}=\left(\left\|v_{0 m}\right\|_{0}^{2}+\alpha a\left(v_{0 m}, v_{0 m}\right)\right)^{1 / 2}$. Hence

$$
\left\|\sum_{i=1}^{N} \eta_{i} v_{m}\left(T_{i}\right)\right\|_{*} \leq \sum_{i=1}^{N}\left\|\eta_{i} v_{m}\left(T_{i}\right)\right\|_{*}=\sum_{i=1}^{N}\left|\eta_{i}\right|\left\|v_{m}\left(T_{i}\right)\right\|_{*} \leq \sum_{i=1}^{N}\left|\eta_{i}\right| \rho \leq \rho .
$$

Let $\bar{B}_{m}(\rho)=\left\{v_{0 m} \in W_{m}:\left\|v_{0 m}\right\|_{*} \leq \rho\right\}$ be a closed ball in the space $W_{m}$. Let us define

$$
\begin{aligned}
\mathcal{F}_{m}: \bar{B}_{m}(\rho) & \rightarrow \bar{B}_{m}(\rho) \\
v_{0 m} & \longmapsto \mathcal{F}_{m}\left(v_{0 m}\right)=\sum_{i=1}^{N} \eta_{i} v_{m}\left(T_{i}\right) .
\end{aligned}
$$

We prove that $\mathcal{F}_{m}$ is a contraction. Let $v_{0 m}, \bar{v}_{0 m} \in \bar{B}_{m}(\rho)$ and let $y_{m}(t)=v_{m}(t)-\bar{v}_{m}(t)$, where $v_{m}(t)$ and $\bar{v}_{m}(t)$ are solutions of the system (3.7) on [0,T] satisfying the initial conditions $v_{m}(0)=v_{0 m}$ and $\bar{v}_{m}(0)=\bar{v}_{0 m}$, respectively. Then $y_{m}(t)$ satisfies the following differential equation system:

$$
\begin{aligned}
& \left\langle y_{m}^{\prime}(t), w_{j}\right\rangle+\alpha a\left(y_{m}^{\prime}(t), w_{j}\right)+\mu a\left(y_{m}(t), w_{j}\right) \\
& \quad+\left\langle f\left(v_{m}(t)+\varphi(t)\right)-f\left(\bar{v}_{m}(t)+\varphi(t)\right), w_{j}\right\rangle=0,
\end{aligned}
$$

$1 \leq j \leq m$, with the initial condition

$$
y_{m}(0)=v_{0 m}-\bar{v}_{0 m} \text {. }
$$


By using the same arguments as before, we can show that

$$
\frac{d}{d t}\left[\left\|y_{m}(t)\right\|_{0}^{2}+\alpha a\left(y_{m}(t), y_{m}(t)\right)\right]+2 \bar{\gamma}\left[\left\|y_{m}(t)\right\|_{0}^{2}+\alpha a\left(y_{m}(t), y_{m}(t)\right)\right] \leq 0
$$

where $\bar{\gamma}=\beta_{1}\left[\mu-\delta \frac{R}{2}(R-1)^{2}\right]>0, \beta_{1}=\frac{1}{2} \min \left\{\frac{1}{\alpha}, \frac{2}{R(R-1)^{2}}\right\}$.

Integrating the inequality (5.19), we obtain

$$
\left\|y_{m}(t)\right\|_{*} \leq e^{-\bar{\gamma} t}\left\|v_{0 m}-\bar{v}_{0 m}\right\|_{*} \quad \text { for all } t \in[0, T]
$$

hence

$$
\begin{aligned}
\left\|\sum_{i=1}^{N} \eta_{i} y_{m}\left(T_{i}\right)\right\|_{*} & \leq \sum_{i=1}^{N}\left\|\eta_{i} y_{m}\left(T_{i}\right)\right\|_{*}=\sum_{i=1}^{N}\left|\eta_{i}\right|\left\|y_{m}\left(T_{i}\right)\right\|_{*} \\
& \leq \sum_{i=1}^{N}\left|\eta_{i}\right| e^{-\bar{\gamma} T_{i}}\left\|v_{0 m}-\bar{v}_{0 m}\right\|_{*} \leq e^{-\bar{\gamma} T_{1}}\left\|v_{0 m}-\bar{v}_{0 m}\right\|_{*},
\end{aligned}
$$

or

$$
\left\|\mathcal{F}_{m}\left(v_{0 m}\right)-\mathcal{F}_{m}\left(\bar{v}_{0 m}\right)\right\|_{*} \leq e^{-\bar{\gamma} T_{1}}\left\|v_{0 m}-\bar{v}_{0 m}\right\|_{*},
$$

i.e., $\mathcal{F}_{m}$ is a contraction.

Therefore, there exists a unique function $v_{0 m} \in \bar{B}_{m}(\rho)$ such that the solution of the initial value problem (3.7) is a solution of the system $(3.7)_{1},(5.4)$. This solution satisfies the inequality (5.13) a.e., in $[0, T]$.

On the other hand, we multiplying the $j$ th equation of (3.7) by $c_{m j}^{\prime}(t)$ and summing up with respect to $j$, afterward integrating with respect to the time variable from 0 to $T$, we get after some rearrangements

$$
\begin{aligned}
& 2 \int_{0}^{T}\left(\left\|v_{m}^{\prime}(t)\right\|_{0}^{2}+\alpha a\left(v_{m}^{\prime}(t), v_{m}^{\prime}(t)\right)\right) d t+\mu \int_{0}^{T} \frac{d}{d t} a\left(v_{m}(t), v_{m}(t)\right) d t \\
& \quad=2 \int_{0}^{T}\left\langle f_{2}(t), v_{m}^{\prime}(t)\right\rangle d t-2 \int_{0}^{T}\left\langle f\left(v_{m}(t)+\varphi(t)\right), v_{m}^{\prime}(t)\right\rangle d t .
\end{aligned}
$$

From (5.13), we obtain

$$
\begin{aligned}
&\left|\int_{0}^{T} \frac{d}{d t} a\left(v_{m}(t), v_{m}(t)\right) d t\right|=\left|a\left(v_{m}(T), v_{m}(T)\right)-a\left(v_{m}(0), v_{m}(0)\right)\right| \\
& \leq a\left(v_{m}(T), v_{m}(T)\right)+a\left(v_{m}(0), v_{m}(0)\right) \\
& \leq \frac{1}{\alpha}\left[\left\|v_{m}(T)\right\|_{*}^{2}+\left\|v_{m}(0)\right\|_{*}^{2}\right] \leq \frac{1}{\alpha} \rho^{2} ; \\
& 2 \int_{0}^{T}\left\langle f_{2}(t), v_{m}^{\prime}(t)\right\rangle d t \leq 2 \int_{0}^{T}\left\|f_{2}(t)\right\|_{0}^{2} d t+\frac{1}{2} \int_{0}^{T}\left\|v_{m}^{\prime}(t)\right\|_{0}^{2} d t .
\end{aligned}
$$

Note that

$$
\|\varphi(t)\|_{C^{0}([1, R])} \leq\left[R+h_{1}(R-1)\right] \sup _{0 \leq t \leq T}\left(\left|g_{1}(t)\right|+\left|g_{R}(t)\right|\right)
$$


and

$$
\begin{aligned}
\left\|v_{m}(t)+\varphi(t)\right\|_{C^{0}([1, R])} & \leq\left\|v_{m}(t)\right\|_{C^{0}([1, R])}+\|\varphi(t)\|_{C^{0}([1, R])} \\
& \leq \sqrt{R-1} \frac{\rho}{\sqrt{\alpha}}+\left[R+h_{1}(R-1)\right] \sup _{0 \leq t \leq T}\left(\left|g_{1}(t)\right|+\left|g_{R}(t)\right|\right) \\
& =M_{1}(T) .
\end{aligned}
$$

Hence

$$
\left|f\left(v_{m}(x, t)+\varphi(x, t)\right)\right| \leq \sup _{|z| \leq M_{1}(T)}|f(z)| .
$$

This implies

$$
\begin{aligned}
-2 \int_{0}^{T}\left\langle f\left(v_{m}(t)+\varphi(t)\right), v_{m}^{\prime}(t)\right\rangle d t & \leq 2 \int_{0}^{T}\left\|f\left(v_{m}(t)+\varphi(t)\right)\right\|_{0}^{2} d t+\frac{1}{2} \int_{0}^{T}\left\|v_{m}^{\prime}(t)\right\|_{0}^{2} d t \\
& \leq M_{2}(T)+\frac{1}{2} \int_{0}^{T}\left\|v_{m}^{\prime}(t)\right\|_{0}^{2} d t
\end{aligned}
$$

where $M_{2}(T)=T\left(R^{2}-1\right) \sup _{|z| \leq M_{1}} f^{2}(z)$.

It follows from (5.22), (5.23), (5.24), and (5.28) that

$$
\int_{0}^{T}\left(\left\|v_{m}^{\prime}(t)\right\|_{0}^{2}+\alpha a\left(v_{m}^{\prime}(t), v_{m}^{\prime}(t)\right)\right) d t \leq \frac{1}{\alpha} \mu \rho^{2}+M_{2}(T)+2 \int_{0}^{T}\left\|f_{2}(t)\right\|_{0}^{2} d t \leq C_{T}
$$

for all $m \in \mathbb{N}$, for all $t \in[0, T]$, where $C_{T}$ always indicates a bound depending on $T$.

Step 3 . The limiting process.

By (5.13) and (5.29) we deduce that there exists a subsequence of $\left\{v_{m}\right\}$, still denoted by $\left\{v_{m}\right\}$ such that

$$
\left\{\begin{array}{l}
v_{m} \rightarrow v \quad \text { in } L^{\infty}(0, T ; V) \text { weak* } \\
v_{m}^{\prime} \rightarrow v^{\prime} \quad \text { in } L^{2}(0, T ; V) \text { weak. }
\end{array}\right.
$$

From (5.4), we obtain

$$
v(0)=\sum_{i=1}^{N} \eta_{i} v\left(T_{i}\right) .
$$

Indeed, we prove (5.31) as follows.

By $\left\|v_{m}(0)\right\|_{*}=\left\|v_{0 m}\right\|_{*} \leq \rho$, and the imbedding $V \hookrightarrow C^{0}(\bar{\Omega})$ is compact, there exists a subsequence of $\left\{v_{0 m}\right\}$, still denoted by $\left\{v_{0 m}\right\}$ such that

$$
\begin{cases}v_{0 m} \rightarrow \tilde{v}_{0} & \text { in } V \text { weakly, } \\ v_{0 m} \rightarrow \tilde{v}_{0} & \text { in } C^{0}([1, R]) \text { strongly. }\end{cases}
$$

From the equality $v_{m}(t)=v_{m}(0)+\int_{0}^{t} v_{m}^{\prime}(s) d s$, we deduce from (5.30) and (5.32) that

$$
v(t)=\tilde{v}_{0}+\int_{0}^{t} v^{\prime}(s) d s
$$


This implies $v(0)=\tilde{v}_{0}$ and

$$
\left\{\begin{array}{l}
v_{0 m} \rightarrow v(0) \quad \text { in } V \text { weakly, } \\
v_{0 m} \rightarrow v(0) \quad \text { in } C^{0}([1, R]) \text { strongly. }
\end{array}\right.
$$

From (5.4), we obtain

$$
\begin{aligned}
\left\langle v_{m}(0), w_{j}\right\rangle & =\sum_{i=1}^{N} \eta_{i}\left\langle v_{m}\left(T_{i}\right), w_{j}\right\rangle \\
& =\sum_{i=1}^{N} \eta_{i}\left[\left\langle v_{m}(0), w_{j}\right\rangle+\int_{0}^{T_{i}}\left\langle v_{m}^{\prime}(t), w_{j}\right\rangle d t\right], \quad \forall j \in \mathbb{N} .
\end{aligned}
$$

By (5.30), (5.34) and (5.35), we deduce that

$$
\left\langle v(0), w_{j}\right\rangle=\sum_{i=1}^{N} \eta_{i}\left[\left\langle v(0), w_{j}\right\rangle+\int_{0}^{T_{i}}\left\langle v^{\prime}(t), w_{j}\right\rangle d t\right]=\sum_{i=1}^{N} \eta_{i}\left\langle v\left(T_{i}\right), w_{j}\right\rangle, \quad \forall j \in \mathbb{N} .
$$

Hence $v(0)=\sum_{i=1}^{N} \eta_{i} v\left(T_{i}\right)$, therefore, (5.31) is proved.

Using a compactness lemma ([19], Lions, p.57) applied to (5.30), we can extract from the sequence $\left\{v_{m}\right\}$ a subsequence, still denoted by $\left\{v_{m}\right\}$, such that

$$
v_{m} \rightarrow v \quad \text { strongly in } L^{2}\left(Q_{T}\right) .
$$

By the Riesz-Fischer theorem, we can extract from $\left\{v_{m}\right\}$ a subsequence, still denoted by $\left\{v_{m}\right\}$, such that

$$
v_{m}(x, t) \rightarrow v(x, t) \quad \text { a.e. }(x, t) \text { in } Q_{T} .
$$

Because $f$ is continuous, we have

$$
f\left(v_{m}(x, t)+\varphi(x, t)\right) \rightarrow f(v(x, t)+\varphi(x, t)) \quad \text { a.e. }(x, t) \text { in } Q_{T} .
$$

Using the dominated convergence theorem, (5.27) and (5.38) yield

$$
f\left(v_{m}+\varphi\right) \rightarrow f(v+\varphi) \quad \text { strongly in } L^{2}\left(Q_{T}\right) .
$$

Denote by $\left\{\zeta_{i}, i=1,2, \ldots\right\}$ the orthonormal base in the real Hilbert space $L^{2}(0, T)$. The set $\left\{\zeta_{i} w_{j}, i, j=1,2, \ldots\right\}$ forms an orthonormal base in $L^{2}(0, T ; V)$. From (3.7) we have

$$
\begin{gathered}
\int_{0}^{T}\left\langle v_{m}^{\prime}(t), w_{j} \zeta_{i}(t)\right\rangle d t+\alpha \int_{0}^{T} a\left(v_{m}^{\prime}(t), w_{j} \zeta_{i}(t)\right) d t+\mu \int_{0}^{T} a\left(v_{m}(t), w_{j} \zeta_{i}(t)\right) d t \\
\quad+\int_{0}^{T}\left\langle f\left(v_{m}(t)+\varphi(t)\right), w_{j} \zeta_{i}(t)\right\rangle d t=\int_{0}^{T}\left\langle f_{2}(t), w_{j} \zeta_{i}(t)\right\rangle d t
\end{gathered}
$$

for all $i, j, 1 \leq j \leq m, i \in \mathbb{N}$. 
For $i, j$ fixed, we deduce from (5.39) that

$$
\int_{0}^{T}\left\langle f\left(v_{m}(t)+\varphi(t)\right), w_{j} \zeta_{i}(t)\right\rangle d t \rightarrow \int_{0}^{T}\left\langle f(v(t)+\varphi(t)), w_{j} \zeta_{i}(t)\right\rangle d t
$$

Passing to the limit in (5.40) by (5.30), (5.41), we obtain

$$
\begin{gathered}
\int_{0}^{T}\left\langle v^{\prime}(t), w_{j} \zeta_{i}(t)\right\rangle d t+\alpha \int_{0}^{T} a\left(v^{\prime}(t), w_{j} \zeta_{i}(t)\right) d t+\mu \int_{0}^{T} a\left(v(t), w_{j} \zeta_{i}(t)\right) d t \\
+\int_{0}^{T}\left\langle f(v(t)+\varphi(t)), w_{j} \zeta_{i}(t)\right\rangle d t=\int_{0}^{T}\left\langle f_{2}(t), w_{j} \zeta_{i}(t)\right\rangle d t .
\end{gathered}
$$

Equation (5.42) holds for every $i, j \in \mathbb{N}$, i.e., the equation

$$
\begin{aligned}
& \int_{0}^{T}\left\langle v^{\prime}(t), w(t)\right\rangle d t+\alpha \int_{0}^{T} a\left(v^{\prime}(t), w(t)\right) d t+\mu \int_{0}^{T} a(v(t), w(t)) d t \\
& \quad+\int_{0}^{T}\langle f(v(t)+\varphi(t)), w(t)\rangle d t=\int_{0}^{T}\left\langle f_{2}(t), w(t)\right\rangle d t \quad \text { for all } w \in L^{2}(0, T ; V)
\end{aligned}
$$

is fulfilled.

Step 4. Uniqueness of the solutions.

Assume now that $N=1$ is satisfied. Let $v_{1}$ and $v_{2}$ be two solutions of (5.2). Then $v=v_{1}-v_{2}$ satisfies the following problem:

$$
\left\{\begin{array}{l}
\int_{0}^{T}\left\langle v^{\prime}(t), w(t)\right\rangle d t+\alpha \int_{0}^{T} a\left(v^{\prime}(t), w(t)\right) d t+\mu \int_{0}^{T} a(v(t), w(t)) d t \\
\quad+\int_{0}^{T}\left\langle f\left(v_{1}(t)+\varphi(t)\right)-f\left(v_{2}(t)+\varphi(t)\right), w(t)\right\rangle d t=0, \quad \forall w \in L^{2}(0, T ; V), \\
v(0)=\eta_{N} v(T), \quad\left|\eta_{N}\right| \leq 1 \\
v \in L^{\infty}(0, T ; V), \quad v_{t} \in L^{2}(0, T ; V) .
\end{array}\right.
$$

Taking $w=v$ in $(5.44)_{1}$ and using $(5.44)_{2}$, we get

$$
\begin{aligned}
\int_{0}^{T}\left\langle v^{\prime}(t), v(t)\right\rangle d t= & \frac{1}{2}\|v(T)\|_{0}^{2}-\frac{1}{2}\|v(0)\|_{0}^{2}=\frac{1}{2}\left(1-\eta_{N}^{2}\right)\|v(T)\|_{0}^{2} \geq 0 \\
\int_{0}^{T} a\left(v^{\prime}(t), v(t)\right) d t & =\frac{1}{2} a(v(T), v(T))-\frac{1}{2} a(v(0), v(0)) \\
& =\frac{1}{2}\left(1-\eta_{N}^{2}\right) a(v(T), v(T)) \geq 0 .
\end{aligned}
$$

Hence

$$
\begin{aligned}
\mu \int_{0}^{T} a(v(t), v(t)) d t & \leq-\int_{0}^{T}\left\langle f\left(v_{1}(t)+\varphi(t)\right)-f\left(v_{2}(t)+\varphi(t)\right), v(t)\right\rangle d t \\
& \leq \delta \int_{0}^{T}\|v(t)\|_{0}^{2} d t \leq \delta \frac{R}{2}(R-1)^{2} \int_{0}^{T}\left\|v_{x}(t)\right\|_{0}^{2} d t \\
& \leq \delta \frac{R}{2}(R-1)^{2} \int_{0}^{T} a(v(t), v(t)) d t
\end{aligned}
$$

By $0<\delta<\frac{2 \mu}{R(R-1)^{2}}$, implies $\delta \frac{R}{2}(R-1)^{2}<\mu$, we deduce from (5.46) that $\int_{0}^{T} a(v(t), v(t)) d t=$ 0, i.e., $v=v_{1}-v_{2}=0$.

This completes the proof of Theorem 5.1. 


\section{Competing interests}

The authors declare that they have no competing interests.

\section{Authors' contributions}

All authors contributed equally in this article. They read and approved the final manuscript.

\section{Author details}

${ }^{1}$ University of Khanh Hoa, 01 Nguyen Chanh Str., Nha Trang City, Vietnam. ${ }^{2}$ The Faculty of Natural Basic Sciences, Vietnamese Naval Academy, 30 Tran Phu Str., Nha Trang City, Vietnam. ${ }^{3}$ Department of Mathematics and Computer Science, University of Natural Science, Vietnam National University Ho Chi Minh City, 227 Nguyen Van Cu Str., Dist. 5, Ho Chi Minh City, Vietnam. ${ }^{4}$ Department of Mathematics, University of Economics of Ho Chi Minh City, $59 \mathrm{C}$ Nguyen Dinh Chieu Str., Dist. 3, Ho Chi Minh City, Vietnam.

\section{Acknowledgements}

The authors wish to express their sincere thanks to the editor and the referees for the suggestions, remarks and valuable comments.

\section{Received: 6 February 2016 Accepted: 21 July 2016 Published online: 28 July 2016}

\section{References}

1. Al'shin, AB, Korpusov, MO, Sveshnikov, AG: Blow-up in Nonlinear Sobolev Type Equations. de Gruyter Series in Nonlinear Analysis and Applications, vol. 15. de Gruyter, Berlin (2011)

2. Carroll, RW, Showalter, RE: Singular and Degenerate Cauchy Problems. Mathematics in Science and Engineering, vol. 127. Academic Press, New York (1976)

3. Showalter, RE, Ting, TW: Asymptotic behavior of solutions of pseudo-parabolic partial differential equations. Ann. Mat. Pura Appl. 90(4), 241-258 (1971)

4. Showalter, RE: Existence and representation theorems for a semilinear Sobolev equation in Banach space. SIAM J. Math. Anal. 3, 527-543 (1972)

5. Mahmood, A, Khan, NA, Fetecau, C, Jamil, M, Rubbab, Q: Exact analytic solutions for the flow of second grade fluid between two longitudinally oscillating cylinders. J. Prime Res. Math. 5, 192-204 (2009)

6. Asghar, S, Hayat, T, Ariel, PD: Unsteady Couette flows in a second grade fluid with variable material properties Commun. Nonlinear Sci. Numer. Simul. 14(1), 154-159 (2009)

7. Hayat, T, Khan, M, Siddiqui, AM, Asghar, S: Transient flows of a second grade fluid. Int. J. Non-Linear Mech. 39 1621-1633 (2004)

8. Hayat, T, Khan, M, Ayub, M, Siddiqui, AM: The unsteady Couette flow of a second grade fluid in a layer of porous medium. Arch. Mech. 57(5), 405-416 (2005)

9. Hayat, T, Khan, M, Ayub, M: Some analytical solutions for second grade fluid flows for cylindrical geometries. Math. Comput. Model. 43(1-2), 16-29 (2006)

10. Hayat, T, Hussain, M, Khan, M: Hall effect on flows of an Oldroyd-B fluid through porous medium for cylindrical geometries. Comput. Math. Appl. 52(3-4), 269-282 (2006)

11. Hayat, T, Sajid, M, Ayub, M: On explicit analytic solution for MHD pipe flow of a fourth grade fluid. Commun. Nonlinear Sci. Numer. Simul. 13(4), 745-751 (2008)

12. Khan, M, Naheed, E, Fetecau, C, Hayat, T: Exact solutions of starting flows for second grade fluid in a porous medium. Int. J. Non-Linear Mech. 43(9), 868-879 (2008)

13. Khan, M, Asghar, S, Hayat, T: Hall effect on the pipe flow of a Burgers' fluid: an exact solution. Nonlinear Anal., Real World Appl. 10(2), 974-979 (2009)

14. Long, NT, Dinh, APN: On a nonlinear parabolic equation involving Bessel's operator associated with a mixed inhomogeneous condition. J. Comput. Appl. Math. 196(1), 267-284 (2006)

15. Ngoc, LTP, Dinh, NVY, Alain, PN, Long, NT: On a nonlinear heat equation associated with Dirichlet-Robin conditions. Numer. Funct. Anal. Optim. 33(2), 166-189 (2012)

16. Cordier, S, Truong, LX, Long, NT, Dinh, APN: Large time behavior of differential equations with drifted periodic coefficients modeling carbon storage in soil. Appl. Math. Comput. 218(9), 5641-5654 (2012)

17. Truong, LX, Ngoc, LTP, Hoa, CH, Long, NT: On a system of nonlinear wave equations associated with the helical flows of Maxwell fluid. Nonlinear Anal., Real World Appl. 12(6), 3356-3372 (2011)

18. Showalter, RE: Hilbert space methods for partial differential equations. Electron. J. Differ. Equ., Monograph 01 (1994)

19. Lions, JL: Quelques méthodes de résolution des problèmes aux limites nonlinéaires. Dunod, Paris (1969) 